Pair-production of charged Dirac particles on charged Nariai and ultracold black hole manifolds

This content has been downloaded from IOPscience. Please scroll down to see the full text. JHEP08(2009)028

(http://iopscience.iop.org/1126-6708/2009/08/028)

View the table of contents for this issue, or go to the journal homepage for more

Download details:

IP Address: 130.186.11.41

This content was downloaded on 15/11/2016 at 20:09

Please note that terms and conditions apply.

You may also be interested in:

Relativistic extension of shape-invariant potentials

A D Alhaidari

Quantum instability for charged scalar particles on Nariai and black hole manifolds

F Belgiorno, S L Cacciatori and F Dalla Piazza

Production of Dirac particles in vacuum

M N Hounkonnou and M Naciri

Ehrenfest-Type Theorems for a One-Dimensional Dirac Particle

Vidal Alonso and Salvatore De Vincenzo

On particles tunneling from the Taub-NUT-AdS black hole

Zeng Xiao-Xiong and Li Qiang

Tunneling of Dirac Particles from Kaluza-Klein Black Hole

Zeng Xiao-Xiong and Li Qiang

Absorption Cross Section of Einstein-Maxwell Dilation Axion BlackHole for Dirac Particles

Liu Chang-Qing and Jing Ji-Liang 


\title{
Pair-production of charged Dirac particles on charged Nariai and ultracold black hole manifolds
}

\author{
F. Belgiorno, ${ }^{a}$ S.L. Cacciatori ${ }^{b, c}$ and F. Dalla Piazza ${ }^{b, c}$ \\ ${ }^{a}$ Dipartimento di Matematica, Universita' degli Studi di Milano, \\ Via Saldini 50, 20133 Milano, Italy \\ ${ }^{b}$ Dipartimento di Fisica e Matematica, Universita' dell'Insubria, \\ Via Valleggio 11, 22100 Como, Italy \\ ${ }^{c}$ I.N.F.N., Sezione di Milano, \\ Via Celoria 16, 20133 Milano, Italy \\ E-mail: belgiorno@mi.infn.it, sergio.cacciatori@uninsubria.it, \\ f.dallapiazza@uninsubria.it
}

ABSTRACT: Spontaneous loss of charge by charged black holes by means of pair-creation of charged Dirac particles is considered. We provide three examples of exact calculations for the spontaneous discharge process for $4 \mathrm{D}$ charged black holes by considering the process on three special non-rotating de Sitter black hole backgrounds, which allow to bring back the problem to a Kaluza-Klein reduction. Both the zeta-function approach and the transmission coefficient approach are taken into account. A comparison between the two methods is also provided, as well as a comparison with WKB results. In the case of non-zero temperature of the geometric background, we also discuss thermal effects on the discharge process.

KeYwords: Nonperturbative Effects, Thermal Field Theory, Black Holes

ARXIV EPRINT: 0906.1520 


\section{Contents}

1 Introduction $\quad 1$

2 Vacuum instability and thermal states 3

2.1 Vacuum instability in the transmission coefficient approach 3

2.2 Finite temperature effects 5

3 Ultracold II case $\quad 8$

3.1 The transmission coefficient approach 8

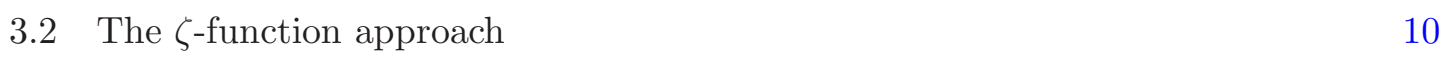

4 The ultracold I case $\quad 13$

$\begin{array}{ll}\text { 4.1 The transmission coefficient approach } & 13\end{array}$

$\begin{array}{lll}4.2 & \text { The } \zeta \text {-function approach } & 16\end{array}$

$\begin{array}{lll}4.3 & \text { Instability of the thermal state } & 19\end{array}$

$\begin{array}{lll}5 & \text { Nariai case } & 19\end{array}$

5.1 The transmission coefficient approach 20

$\begin{array}{lll}5.2 & \text { The } \zeta \text {-function approach } & 24\end{array}$

$\begin{array}{ll}5.3 & \text { Instability of the thermal state } \\ \end{array}$

$\begin{array}{llr}6 & \text { Conclusions } & 29\end{array}$

\section{Introduction}

Spontaneous loss of charge by a charged black hole is a relevant topic in the framework of quantum effects in the field of a black hole $[1,2]$. It belongs to the framework of phenomena which are due to vacuum instability in presence of an external field, with consequent pair creation. Quantum-electrodynamics effects in presence of an external electric field have been in particular a key-topic which has been extensively discussed. Being our interest oriented toward an application to black hole physics, we limit ourselves to quote two seminal papers $[3,4]$ and, in the recent literature, refs. [5-7]. An effective description of pair creation phenomenon for static charged black holes was provided by Damour, Deruelle and Ruffini in a series of papers [8-10]. ${ }^{1}$ To sum up, on these backgrounds the Hamilton-Jacobi equations (H-J) for a classical charged particle can be easily reduced to quadrature by means of variables separation. In particular, the radial equation describes a one dimensional motion of a particle in a given potential. The H-J equation, beyond a positive energy potential,

\footnotetext{
${ }^{1}$ Also rotating black holes were treated therein.
} 
determines a negative energy potential which at classical level must be discarded. However, at quantum level, negative energy states must be included, and a quantum interpretation to this couple of potentials can be given. The positive energy potential determines the allowed positive energy states, whereas the negative energy potential determines the allowed negative energy states. The usual separation of these states occurring in absence of external fields is not ensured a priori, and there can be regions where an overlap of positive and negative states for the particle is allowed, i.e. the Klein paradox takes place. In these level-crossing regions, by means of tunneling between negative and positive states, pair production of charged particles can take place with a rate determined by the transmission probability for the particle to cross the forbidden region between the two potentials, and can be computed e.g. in the WKB approximation.

We improved this semiclassical picture in the case of anti de Sitter Reissner-Nordström black holes showing that the potentials have a direct interpretation at the quantum level without referring to the classical H-J equation [11]. Then, for the class of de Sitter ReissnerNordström black holes we found that level-crossing is always present, due to the peculiar occurrence of both a black hole event horizon and a cosmological event horizon [12], and we also considered a particular limit case, when the black hole horizon radius $r_{+}$equates the cosmological horizon radius $r_{c}$ : the Nariai black hole [13-15]. The aforementioned class of solutions contains further limit cases, corresponding to the extremal cases $r_{-}=r_{+}=r_{c}$, which are called ultracold solutions of type I and II [13, 15]. A careful WKB analysis was also performed for the Nariai case and the ultracold ones.

Herein, we develop our analysis of the pair-creation process associated with the black hole electrostatic field, and fully exploit the fact that the aforementioned special backgrounds allow an exact calculation of the vacuum instability. As a consequence, we can provide for the first time, to our knowledge, exact results for the instability of $4 \mathrm{D}$ charged black holes. We point out that our backgrounds are of a special character: in all the cases the geometry involved is the one of a Cartesian product $M^{1,1} \times S^{2}$ where $M^{1,1}$ is a two dimensional spacetime and $S^{2}$ is a sphere with constant radius (there is not any non-constant warping factor). Moreover, the fluxes do not involve the sphere directions so that the sphere could be effectively considered as an internal space and the problem can be reduced to a two dimensional effective theory by means of a Kaluza-Klein reduction [16-19]. Here we will recall manifestly the strategy of a K-K reduction in part of our analysis. Indeed, for all cases, the Dirac equation will be reduced to a two dimensional Dirac equation on the $M^{1,1}$ background, with the two dimensional spinors obtained by the two dimensional reduction of the four components Dirac spinors and the mass spectra corrected by the K-K modes. We also recall that in a K-K reduction the latter corrections are provided by the harmonic analysis of the internal space $S^{2}$ :

$$
\mu^{2} \longrightarrow \mu_{l}^{2}=\mu^{2}+\lambda_{l}
$$

where $\mu$ is the particle mass and $\lambda_{l}$ are the eigenvalues of the Laplacian operator $-\Delta_{S^{2}}$ on the internal space. In our case we are involved with harmonic analysis for spinors, and then the Dirac "angular momentum" operator eigenvalues $k= \pm\left(j+\frac{1}{2}\right) \in \mathbb{Z}-\{0\}$ appear in $\lambda_{l}$. 
We also take into account the fact that, both in the Nariai case and in the ultracold I one, the real quantum state to be considered is not the Boulware-like state corresponding to standard quantum vacuum, but the Hartle-Hawking state associated with the black hole temperature. Then, we discuss also how pair-creation due to the electrostatic field of the black hole superimposes to the thermal radiation effect which is present in the given backgrounds. We show that, in the thermal means of the Dirac field number operators, a standard thermal contribution appears together with a term which is still related to the aforementioned vacuum instability, except for a further dependence on the background temperature which is induced by the Hawking effect.

The plan of the paper is the following. In section 2 we recall the transmission coefficient approach to vacuum instability, and consider also how the instability affects thermal states in the framework of Thermofield Dynamics. In section 3 we discuss the ultracold II case, both in the transmission coefficient approach and in the zeta function approach. In section 4, an analogous analysis is carried out for the ultracold I case. In section 5 the Nariai case is considered. In this case, the zeta function approach requires a recently developed calculation strategy [20], which is sketched in the present paper. For all the cases a comparison with WKB results is done. In section 6 conclusions appear.

\section{Vacuum instability and thermal states}

In this section, we first recall some aspects of the pair creation due to vacuum instability, in particular we focus on the so-called transmission coefficient approach to the evaluation of the instability, which is associated with the presence of an imaginary part of the effective action [4], which in the following is approached also by means of $\zeta$-function techniques. Then we consider how instability in external fields affects thermal states, referring to Thermofield Dynamics approach for a general fermionic case, although our interest is in the black hole case.

\subsection{Vacuum instability in the transmission coefficient approach}

There are several ways one can deal with vacuum instability. One consists in Schwinger's approach $[4,21,22]$, with calculations carried out in the correct space-time signature, or in its $\zeta$-function variant where calculations are developed in Euclidean signature and then a rotation to real time is performed. As to vacuum instability, we adopt the $\zeta$-function variant, and a double check of our results is also provided by the so-called transmission coefficient approach. We recall shortly some aspects of the latter, in which, following $[8$, $23,24]$, it is also possible to reconstruct the probability of persistence of the vacuum. Let us introduce, for a diagonal scattering process,

$$
n_{i}^{\mathrm{IN}}=R_{i} n_{i}^{\mathrm{OUT}}+T_{i} p_{i}^{\mathrm{OUT}}
$$

where $n_{i}$ stays for a negative energy mode and $p_{i}$ for a positive energy one. $T_{i}$ is the transmission coefficient and $R_{i}$ is the reflection one. Moreover, in [8] one defines

$$
\eta_{i}:=\left|T_{i}\right|^{2}
$$


which coincides with the mean number per unit time and unit volume of created particles [8]. Cf. also $[23,25]$. In the case of fermions, the result is

$$
\left|R_{i}\right|^{2}=1-\eta_{i}
$$

which excludes any superradiant phenomenon for Dirac particles. As thoroughfully discussed in [43], it is crucial to consider group velocity for the asymptotic behavior of the solutions, in order to get a correct physical result. In fact, an erroneous consideration of phase velocity in place of group velocity could easily lead to conclude that superradiance exists also for the Dirac case.

By interpreting à la Stueckelberg the scattering process, one can also obtain

$$
n_{i}^{\mathrm{OUT}}=R_{i}^{-1} n_{i}^{\mathrm{IN}}-R_{i}^{-1} T_{i} p_{i}^{\mathrm{OUT}}
$$

which is interpreted as the scattering of a negative mode incident from the future and which is in part refracted in the past and in part reflected in the future. The new reflection amplitude $-R_{i}^{-1} T_{i}$ is such that the reflection coefficient

$$
\left|R_{i}^{-1} T_{i}\right|^{2}=\frac{\eta_{i}}{1-\eta_{i}}
$$

can be interpreted as the relative probability for the creation of the pair $n_{i}^{\text {OUT }}, p_{i}^{\text {OUT }}$. The absolute probability is obtained by multiplying the relative one times the probability $p_{i, 0}$ to get zero pairs in the channel $i$, and then the probability $p_{i, n}$ of $n$ pair for fermions is

$$
p_{i, n}=p_{i, 0} \frac{\eta_{i}^{n}}{\left(1-\eta_{i}\right)^{n}}
$$

The normalization condition

$$
\sum_{n=0}^{1} p_{i, n}=1
$$

for fermions leads to

$$
p_{i, 0}=1-\eta_{i} .
$$

The persistence of the vacuum is given by

$$
P_{0}=\prod_{i} p_{i, 0}=\exp (-2 \operatorname{Im} W),
$$

and then

$$
2 \operatorname{Im} W=-\sum_{i} \log \left(1-\eta_{i}\right)=\sum_{i} \sum_{k=1}^{\infty} \frac{1}{k} \eta_{i}^{k}
$$

for fermions. For bosons see e.g. [8]. 


\section{$2.2 \quad$ Finite temperature effects}

In the case of ultracold I and Nariai manifolds, one has to take into account that we deal with a black hole manifold endowed with a non-zero temperature. As a consequence, we have to consider quantum instability not simply for a vacuum state which corresponds to the Boulware-like state of standard Schwarzschild solution, but for the state which plays the role of Hartle-Hawking state for the given solution. Since the discover of the Hawking effect, a very fine construction of the thermal state living on a finite temperature black hole manifold characterized by a bifurcate Killing horizon was introduced by Israel [26] on the grounds of Unruh analysis [27] and of the thermofield approach to thermal physics introduced by Takahashi and Umezawa [28-30]. Israel discovered that the HH state (hence called also Hartle-Hawking-Israel state) corresponds to the thermal vacuum of thermofield approach with the temperature equal to the black-hole temperature, and that the would-be fictitious states of the thermofield approach correspond to the states in the left wedge of the extended solution (if one is living in the right wedge). In our case, one possibility is to consider an analogous construction; alternatively, we adopt a more "liberal" attitude, in the sense that we appeal to Thermofield Dynamics formalism and describe the equilibrium state (KMS state) without caring about the reality of the "would-be fictitious" states in a "specular wedge" of the extended manifold. One could also appeal to the approach developed in [31] (where a KMS quantization, leading to a KMS state, is introduced, without any doubling of the Hilbert space).

We show that the transmission coefficient approach at finite temperature is still a valid approach to analyze the quantum instability problem at hand. Thermofield dynamics also helps a straightforward generalization of quantum instability to the case where the initial (in) and the final (out) states are thermal states (at the same temperature) instead than vacuum ones. We could as well start from results given in [32, 33] and also in [25, 34], which analyze stability topics in quantum electrodynamics. In order to check if there is instability in the thermal state at the Hawking temperature, we adopt the following "standard" strategy: we calculate the mean number in the "in" thermal state of "out" particles in the $l$-mode, and see if there exists any deviation from a purely thermal distribution. Equivalently, we could evaluate the thermal mean of the number of "out" particles in the $l$-mode minus the number of "in" particles in the l-mode (cf. [32]) and then see the net effect of a possible quantum instability. In what follows, our focus is to the case of black hole backgrounds with a single temperature, and then $\beta$ is to be meant in the black hole case as the inverse black hole temperature. Still, we point out that the following analysis holds true for fermions in a generic thermal state with inverse temperature $\beta$.

We refer both to [8] and to [32], and first we consider the Bogoliubov transformation for the "diagonal" case (we purposefully choose a notation which allows a straightforward comparison with [32]; see also [8]):

$$
\begin{aligned}
& a_{l}^{\text {out }}=\mu_{l} a_{l}^{\text {in }}+\nu_{l}\left(b_{l}^{\text {in }}\right)^{\dagger} \\
& b_{l}^{\text {out }}=\mu_{l} b_{l}^{\text {in }}-\nu_{l}\left(a_{l}^{\text {in }}\right)^{\dagger},
\end{aligned}
$$

where $l$ is a collective index specifying states in the Hilbert space, and where the usual 
CCR rules for fermions lead to

$$
\left|\mu_{l}\right|^{2}+\left|\nu_{l}\right|^{2}=1
$$

We are interested in the following operator:

$$
\begin{aligned}
N_{l}^{\text {out }}(a): & =\left(a_{l}^{\text {out }}\right)^{\dagger} a_{l}^{\text {out }} \\
& =\left|\mu_{l}\right|^{2}\left(a_{l}^{\text {in }}\right)^{\dagger} a_{l}^{\text {in }}+\nu^{*} \mu b_{l}^{\text {in }} a_{l}^{\text {in }}+\mu^{*} \nu\left(a_{l}^{\text {in }}\right)^{\dagger}\left(b_{l}^{\text {in }}\right)^{\dagger}+\left|\nu_{l}\right|^{2}\left(1-\left(b_{l}^{\text {in }}\right)^{\dagger} b_{l}^{\text {in }}\right) .
\end{aligned}
$$

For our aims, it works equally well the operator which allows to detect the net effect of the instability (cf. [32])

$$
\bar{N}_{l}^{\text {out }}(a):=\left(a_{l}^{\text {out }}\right)^{\dagger} a_{l}^{\text {out }}-\left(a_{l}^{\text {in }}\right)^{\dagger} a_{l}^{\text {in }} .
$$

We also introduce thermal state operators, according to the standard constructions in thermofield dynamics $[29,30,35]$, both for initial and final states. We omit "in" and "out" labels in this case, for simplicity of notation, and introduce the thermal state $\mid O(\beta)>$ and thermal state annihilation operators $a_{l}(\beta), \tilde{a}_{l}(\beta), b_{l}(\beta), \tilde{b}_{l}(\beta)$, which are such that

$$
a_{l}(\beta)\left|O(\beta)>=\tilde{a}_{l}(\beta)\right| O(\beta)>=b_{l}(\beta)\left|O(\beta)>=\tilde{b}_{l}(\beta)\right| O(\beta)>=0 .
$$

We are mainly interested in the following relations (see also [36]):

$$
\begin{aligned}
& a_{l}=s_{l}^{+} a_{l}(\beta)+c_{l}^{+} \tilde{a}_{l}^{\dagger}(\beta), \\
& b_{l}=s_{l}^{-} b_{l}(\beta)+c_{l}^{-} \tilde{b}_{l}^{\dagger}(\beta),
\end{aligned}
$$

with

$$
\begin{aligned}
c_{l}^{+}: & =\frac{1}{\sqrt{1+\exp \left[\beta\left(\omega-\varphi^{+}\right)\right]}}, \\
s_{l}^{+}: & =\frac{\exp \left[\frac{1}{2} \beta\left(\omega-\varphi^{+}\right)\right]}{\sqrt{1+\exp \left[\beta\left(\omega-\varphi^{+}\right)\right]}},
\end{aligned}
$$

and the analogous ones for $b$-operators (which correspond to operators for antiparticles, i.e. for negative frequency states; cf. [29]):

$$
\begin{aligned}
c_{l}^{-}: & =\frac{1}{\sqrt{1+\exp \left[\beta\left(|\omega|+\varphi^{-}\right)\right]}}, \\
s_{l}^{-}: & =\frac{\exp \left[\frac{1}{2} \beta\left(|\omega|+\varphi^{-}\right)\right]}{\sqrt{1+\exp \left[\beta\left(|\omega|+\varphi^{-}\right)\right.}},
\end{aligned}
$$

where $\varphi^{+}, \varphi^{-}$are chemical potentials for particles and antiparticles respectively [36].

In the previous formula and in the following ones, for simplicity of notation we make a "liberal" use of indexes for quantum numbers, as far as unambiguous formulas arise. By making use of the above relations between particle operators and thermal state creation and annihilation operators, we easily find for particles

$$
<\bar{N}_{l}^{\text {out }}>_{\beta}=\left|\nu_{l}\right|^{2}\left(1-\left(c_{l}^{+}\right)^{2}-\left(c_{l}^{-}\right)^{2}\right)=\left|\nu_{l}\right|^{2} \frac{1}{2}\left(\tanh \left[\frac{1}{2} \beta\left(\omega-\varphi^{+}\right)\right]+\tanh \left[\frac{1}{2} \beta\left(|\omega|+\varphi^{-}\right)\right]\right)
$$


this result in the limit as $\varphi^{+}, \varphi^{-} \rightarrow 0$ agrees with the result displayed in [32]. Note that this means that

$$
<N_{l}^{\text {out }}>_{\beta}=\left(c_{l}^{+}\right)^{2}+\left|\nu_{l}\right|^{2}\left(1-\left(c_{l}^{+}\right)^{2}-\left(c_{l}^{-}\right)^{2}\right)=\frac{1}{1+\exp \left[\beta\left(\omega-\varphi^{+}\right)\right]}+<\bar{N}_{l}^{\text {out }}>_{\beta},
$$

where the former term is the expected mean number of fermions in thermal equilibrium at the given temperature and the latter term is the net effect associated with the pair production induced by the presence of an electrostatic field. In our notation for the the Nariai case, we shall get

$$
<\bar{N}_{k}^{\text {out }}>_{\beta}=\left|T_{k}(\omega)\right|^{2} \frac{1}{2}\left(\tanh \left[\frac{1}{2} \beta\left(\omega-\varphi^{+}\right)\right]+\tanh \left[\frac{1}{2} \beta\left(|\omega|+\varphi^{-}\right)\right]\right)
$$

where $\varphi^{+}$is assumed for definiteness to be the chemical potential for particles in the case of a positively charged black hole, $\varphi^{-}=\varphi^{+}$, particles are electrons (charge $-e$ ) and

$$
\varphi^{+}=-e\left(\left.A_{0}\right|_{\pi}-\left.A_{0}\right|_{0}\right)=-2 e Q \frac{B}{A} .
$$

Formally, an analogous experession holds for the ultracold I case, which is nevertheless pathological (cf. section 4.3). Furthermore, in analogy with Page's analysis in [37], one can expect that charged particles are efficiently emitted with thermal spectrum only for small black hole masses, and then that above the corresponding threshold charged particles are emitted only because of the electrodynamic instability (i.e. as if they were emitted in vacuum).

Moreover, we have to take into account that both in the ultracold I and in the Nariai case we work with dimensionless (rescaled) variables and then we get $\beta=2 \pi$. Note also that

$$
<0 \text { in }\left|N_{k}^{\text {out }}\right| 0 \text { in }>=\left|T_{k}(\omega)\right|^{2} .
$$

It is also interesting to evaluate the following quantity:

$$
\left(\Delta N_{l}\right)^{2}:=<\left(N_{l}^{\text {out }}\right)^{2}>_{\beta}-<N_{l}^{\text {out }}>_{\beta}^{2} .
$$

As to the operator $\left(N_{l}^{\text {out }}\right)^{2}$, being $\left(N_{l}^{\text {out }}\right)^{2}=N_{l}^{\text {out }}$, one finds

$$
\begin{aligned}
\left(\Delta N_{l}\right)^{2}= & <N_{l}^{\text {out }}>_{\beta}\left(1-<N_{l}^{\text {out }}>_{\beta}\right) \\
= & \left(c_{l}^{+}\right)^{2}\left(1-\left(c_{l}^{+}\right)^{2}\right)+\left|\nu_{l}\right|^{2}\left(1-\left|\nu_{l}\right|^{2}\right)-\left|\nu_{l}\right|^{2}\left(1-\left|\nu_{l}\right|^{2}\right)\left[\left(c_{l}^{+}\right)^{2}+\left(c_{l}^{-}\right)^{2}\right] \\
& +\left|\nu_{l}\right|^{4}\left[\left(c_{l}^{+}\right)^{2}+\left(c_{l}^{-}\right)^{2}\right]\left(1-\left(c_{l}^{+}\right)^{2}-\left(c_{l}^{-}\right)^{2}\right)-2\left|\nu_{l}\right|^{2}\left(c_{l}^{+}\right)^{2}\left(1-\left(c_{l}^{+}\right)^{2}-\left(c_{l}^{-}\right)^{2}\right) .
\end{aligned}
$$

In a stable equilibrium situation, i.e. in our case in absence of electrostatic charge, one would obtain

$$
\left(\Delta N_{l}\right)^{2}=\left(c_{l}^{+}\right)^{2}\left(1-\left(c_{l}^{+}\right)^{2}\right),
$$

which corresponds to the first contribution displayed above. Moreover, it is interesting to point out that it holds

$$
<0 \text { in }\left|\left(N_{l}^{\text {out }}\right)^{2}\right| 0 \text { in }>-\left(<0 \text { in }\left|N_{l}^{\text {out }}\right| 0 \text { in }>\right)^{2}=\left|\nu_{l}\right|^{2}\left(1-\left|\nu_{l}\right|^{2}\right),
$$

which amounts to the temperature-independent contribution in the previous formula, where also a third contribution, which "mixes" the pair creation effect to the thermality of the background, occurs. 


\section{Ultracold II case}

The ultracold II metric is obtained from the Reissner-Nordström-de Sitter one in the limit of coincidence of the Cauchy horizon, of the black hole event horizon and of the cosmological event horizon: $r_{-}=r_{+}=r_{c}$. See $[13,15]$. In particular, the metric we are interested in is

$$
d s^{2}=-d t^{2}+d x^{2}+\frac{1}{2 \Lambda}\left(d \theta^{2}+\sin ^{2}(\theta) d \phi^{2}\right),
$$

with $x \in \mathbb{R}$ and $t \in \mathbb{R}$. Then the $(t, x)$-part of the metric is a $2 \mathrm{D}$ Minkowski space, to which a spherical part is warped with a constant warping factor. One gets $\Gamma_{33}^{2}=$ $-\sin (\theta) \cos (\theta), \Gamma_{23}^{3}=\cot (\theta)$. The electromagnetic field strength is $F=-\sqrt{\Lambda} d t \wedge d x$, and we can choose $A_{0}=\sqrt{\Lambda} x$ and $A_{j}=0, j=1,2,3$. It is also useful to define $E:=\sqrt{\Lambda}$, which represents the intensity of the electrostatic field on the given manifold. We note that it is uniform, and then one expects naively to retrieve at least some features of Schwinger's result.

\subsection{The transmission coefficient approach}

Let us consider the reduced Hamiltonian which can be obtained by variable separation as in [12]: starting from the full Dirac equation $(\not D-\mu) \Psi=0$, variable separation leads to the following reduced Hamiltonian:

$$
h_{k}=\left[\begin{array}{cc}
-e \sqrt{\Lambda} x-\mu & \partial_{x}+\sqrt{2 \Lambda} k \\
-\partial_{x}+\sqrt{2 \Lambda} k & -e \sqrt{\Lambda} x+\mu
\end{array}\right] .
$$

$k= \pm\left(j+\frac{1}{2}\right) \in \mathbb{Z}-\{0\}$ is the angular part eigenvalue, $\mu$ and $e$ are the fermion mass and charge respectively. Then one obtains the following equation for $\Phi=e^{-i \omega \psi} \Psi=\left(\begin{array}{c}\phi_{1} \\ \phi_{2}\end{array}\right)$ :

$$
\left[-(e E x+\omega) \mathbb{I}_{2}+i \sigma_{2} \partial_{x}+\sqrt{2 \Lambda} k \sigma_{1}-\mu \sigma_{3}\right] \Phi=0,
$$

where $\sigma_{i}$ are Pauli matrices and $\mathbb{I}_{2}$ is the $2 \times 2$ identity matrix. Let us take the unitary transformation $\Phi=e^{-i \frac{\pi}{4} \sigma_{1}} \xi$ so that $\sigma_{i} \mapsto e^{-i \frac{\pi}{4} \sigma_{1}} \sigma_{1} e^{i \frac{\pi}{4} \sigma_{1}}$ and in particular

$$
\left(\mathbb{I}_{2}, \sigma_{1}, \sigma_{2}, \sigma_{3}\right) \mapsto\left(\mathbb{I}_{2}, \sigma_{1}, \sigma_{3},-\sigma_{2}\right) .
$$

Then one obtains

$$
\left[-(e E x+\omega) \mathbb{I}_{2}+i \sigma_{3} \partial_{x}+\sqrt{2 \Lambda} k \sigma_{1}+\mu \sigma_{2}\right] \xi=0,
$$

which amounts to the following couple of differential equations

$$
\begin{array}{r}
-(e E x+\omega) \xi_{1}+i \partial_{x} \xi_{1}+(\sqrt{2 \Lambda} k-i \mu) \xi_{2}=0, \\
(\sqrt{2 \Lambda} k+i \mu) \xi_{1}-(e E x+\omega) \xi_{2}-i \partial_{x} \xi_{2}=0 .
\end{array}
$$

Then we can get

$$
\xi_{2}=\frac{1}{\sqrt{2 \Lambda} k-i \mu}\left[(e E x+\omega) \xi_{1}-i \partial_{x} \xi_{1}\right]
$$


and the following equation for $\xi_{1}$ is obtained:

$$
\frac{d^{2} \xi_{1}}{d x^{2}}+\left[(e E x+\omega)^{2}+i e E-\mu_{k}^{2}\right] \xi_{1}=0
$$

where $\mu_{k}^{2}:=\mu^{2}+2 \Lambda k^{2}$ is the effective mass corrected by K-K modes. We can define

$$
y=\sqrt{\frac{2}{e E}}(e E x+\omega)
$$

and then we obtain the following equation

$$
\frac{d^{2} \xi_{1}}{d y^{2}}+\left[\frac{1}{4} y^{2}-\left(\frac{\mu_{k}^{2}}{2 e E}-\frac{i}{2}\right)\right] \xi_{1}=0
$$

whose solutions are parabolic cylinder functions [38]. The calculation is completely analogous to the one performed in [8], and as in [8] one can easily show that the transmission coefficient satisfies

$$
\left|T_{k}(\omega)\right|^{2}=\exp \left(-\pi \frac{\mu_{k}^{2}}{e E}\right) .
$$

The latter expression corresponds to the mean number per unit time and unit volume of created pairs and coincides with the WKB approximation for the same coefficient [12]. This means that the WKB approximation is actually exact for the given case. This result is expected, being our case easily realized as a completely analogous to the standard case except for the compact character of the $2 \mathrm{D}$ transverse space. By adopting the strategy in [8], or also the one in [2] for determining the factor preceding the exponential terms, one obtains the following imaginary part of the effective action:

$$
\operatorname{Im} W=-\frac{1}{2} \frac{e E S}{2 \pi} \sum_{k \in \mathbb{Z}-\{0\}} g(k) \log \left(1-\exp \left(-\frac{\pi \mu_{k}^{2}}{e E}\right)\right),
$$

where $g(k)=2(2|k|-1)$ is the degeneracy factor.

A comparison with the standard flat space-time case $[4,41]$ shows that the structure of the ultracold II manifold, which is a product of a 2D flat-spacetime times a 2D sphere, yields to the substitution of the (Gaussian) integral over the transverse dimensions with an infinite sum over $k$, still to be valued. We recall that in the flat space-time case, one finds for the $4 \mathrm{D}$ density of the imaginary part of the effective action

$$
w=2 \frac{(e E)^{2}}{8 \pi^{3}} \sum_{n=1}^{\infty} \frac{1}{n^{2}} \exp \left(-\frac{\pi \mu^{2}}{e E} n\right),
$$

which, by avoiding to perform the integration on the transverse variables $\vec{p}_{\perp}$, becomes

$$
\begin{aligned}
w & =2 \frac{(e E)}{8 \pi^{3}} \int d^{2} \vec{p}_{\perp} \sum_{n=1}^{\infty} \frac{1}{n} \exp \left(-\frac{\pi\left(\mu^{2}+\vec{p}_{\perp}^{2}\right)}{e E} n\right) \\
& =-2 \frac{|e E|}{8 \pi^{3}} \int d^{2} \vec{p}_{\perp} \log \left(1-\exp \left(-\frac{\pi\left(\mu^{2}+\vec{p}_{\perp}^{2}\right)}{e E}\right)\right)
\end{aligned}
$$

which is of course strictly analogous to (3.12). 


\subsection{The $\zeta$-function approach}

We tackle the problem also by the zeta function method. This will be useful later to compare with the more difficult Nariai case. The Euclidean formulation is used. For convenience, we recall that the spectral zeta function associated to an operator $H$ having eigenvalues $\lambda_{n}$ with degeneration $d_{n}$, is defined by

$$
\zeta_{H}(s)=\sum_{n=0}^{\infty} \frac{d_{n}}{\lambda_{n}^{s}}=\frac{1}{\Gamma(s)} \int_{0}^{\infty} x^{s-1} \operatorname{Tr} e^{-H x} d x,
$$

that is substantially the Mellin transform of the $\operatorname{kernel}^{2} K_{H}(x)=\operatorname{Tr} e^{-H x}$. The point is that $-\log \operatorname{det} H=\frac{d}{d s} \zeta_{H}(0)$ defines the Euclidean effective action. After turning back to the Lorentzian signature, the instability is measured by the imaginary part of the effective action, which must thus compared with the vacuum persistence computed with the previous method. To this aim, let us consider the Dirac Euclidean equation for the ultracold II background. The spectrum of the Dirac operator, as well-known, is neither positive definite nor semi-bounded. To overcome this problem, one relates the zeta function of the Dirac operator $\not D$ to the zeta function of its square, and for the sake of completeness we recall some key points. To get the right definition, it is convenient to start with an heuristic reasoning. Let $\Psi$ an eigenfunction of the operator $\not D-\mu$ :

$$
(\not D-\mu) \Psi=\lambda \Psi
$$

Then we have $\left(\lambda_{ \pm}+\mu\right)= \pm \sqrt{I^{2}}$ and thus we can formally write

$\log (\operatorname{det}(\not D-\mu))=\frac{1}{2} \log \left(\operatorname{det}\left(-\mu+\sqrt{D^{2}}\right)\right)+\frac{1}{2} \log \left(\operatorname{det}\left(-\mu-\sqrt{D^{2}}\right)\right)=\frac{1}{2} \log \left(\operatorname{det}\left(\mu^{2}-\not D^{2}\right)\right)$.

The factor $1 / 2$ arises from the double degeneration of each eigenvalue. ${ }^{3}$ Thus, it is convenient to define

$$
-\log (\operatorname{det}(\not D-\mu))=\frac{1}{2} \zeta_{\mu^{2}-\not D^{2}}(0),
$$

and then for the Euclidean effective action we get

$$
W=\frac{1}{2} \zeta_{\mu^{2}-\not D^{2}}^{\prime}(0)
$$

The simplest way to proceed is to compute the eigenvalues of $-\not D^{2}$, and next to add the mass square $\mu^{2}$.

We fully exploit the K-K reduction in order to perform the $\zeta$-function calculation. We first note that for the 4D Dirac operator we have

$$
\not D=: \not H+\not H,
$$

\footnotetext{
${ }^{2}$ We assumed here that the spectrum is strictly positive, but in general this can be weakened by complex analyticity techniques.

${ }^{3}$ If $(\not D-\mu) \Psi_{ \pm}=\lambda_{ \pm} \Psi_{ \pm}$then, for example, $\left(-\mu+\sqrt{D^{2}}\right) \Psi_{ \pm}=\lambda_{+} \Psi_{ \pm}$.
} 
where $\not$ depends only on variables for the first 2D factor of the metric and analogously $F^{\prime}$ depends only on spherical variables of the last 2-sphere factor. When one considers $-D^{2}$, it is easy to realize that one obtains

$$
-D^{2}=-E^{2}-F^{2}
$$

and then the eigenvalue $\lambda^{2}$ of $-D^{2}$ is the sum of the eigenvalue $w^{2}$ of $-E^{2}$ and of the eigenvalue $b^{2} k^{2}$ of $-F^{2}$ ( $b$ is a constant related to the radius of the 2 -sphere factor and $k$ is the usual eigenvalue for the angular momentum operator $K$, which is such that $-F^{2}=b^{2} K^{2}$ ):

$$
\lambda^{2}=w^{2}+b^{2} k^{2} .
$$

In the ultracold cases, one finds $b^{2}=2 \Lambda$; in the Nariai case, one has $b^{2}=B$. Eigenfunctions for $-D^{2}$ (and then also for $-D^{2}+\mu^{2}$ ) are tensor products of eigenfunctions of $-E^{2}$ and of eigenfunctions of $-F^{2}$. As a consequence, degeneracy can be read from the aforementioned tensor product structure.

In what follows, we define $\tilde{\gamma}_{\mu}, \mu=0,1,2,3$, as the Euclidean version of the usual gamma matrices which are such that $\left\{\tilde{\gamma}_{\mu}, \tilde{\gamma}_{\mu}\right\}=2 \delta_{\mu, \nu}$.

In the present case, we obtain the following axpression for $E$ :

$$
\not E=\tilde{\gamma}_{0}\left(\partial_{t}-i e E x\right)+\tilde{\gamma}_{1} \partial_{x},
$$

and then

$$
E^{2}=\left(\partial_{t}-i e E x\right)^{2}+\partial_{x}^{2}+i e E \tilde{\gamma}_{0} \tilde{\gamma}_{1} .
$$

The first two terms are meant to be multiplied by $4 \mathrm{D}$ identity $\mathbb{I}_{4}$. Being $i e E \tilde{\gamma}_{0} \tilde{\gamma}_{1}=$ $e E \sigma_{2} \otimes \mathbb{I}_{2}$, and $\mathbb{I}_{4}=\mathbb{I}_{2} \otimes \mathbb{I}_{2}$, the eigenvalue equation

$$
-E^{2} \psi=w^{2} \psi
$$

is equivalent to the following reduced $2 \mathrm{D}$ equation

$$
\left[-\left(\partial_{t}-i e E x\right)^{2} \mathbb{I}_{2}-\partial_{x}^{2} \mathbb{I}_{2}-e E \sigma_{2}\right] \xi=w^{2} \xi
$$

a further unitary rotation such that $\sigma_{2} \mapsto \sigma_{3}$ carries the problem in a "diagonal" form

$$
\left(-\partial_{x}^{2}-\left(\partial_{t}-i e E x\right)^{2} \mp e E\right) \eta_{ \pm}=w^{2} \eta_{ \pm},
$$

where $\eta_{ \pm}$are components of the $2 \mathrm{D}$ vector eigenfunction of the above eigenvalue problem. Variable separation

$$
\eta_{ \pm}(t, x)=\exp (-i \omega t) \zeta_{ \pm}(x)
$$

leads to the following differential equations:

$$
\partial_{x}^{2} \zeta_{ \pm}+\left[ \pm e E-(\omega+e E x)^{2}+w^{2}\right] \zeta_{ \pm}=0
$$

defining the new variable $z=\frac{(\omega+e E x)}{\sqrt{e E}}$, we get

$$
\frac{d^{2} \zeta_{ \pm}(z)}{d z^{2}}+\left[ \pm 1+\frac{w^{2}}{e E}-z^{2}\right] \zeta_{ \pm}(z)=0
$$


which is easily realized to be the equation one obtains for the standard simple harmonic oscillator. The quantization conditions are

$$
2 n+1= \pm 1-\frac{w^{2}}{e E}
$$

which lead to

$$
\begin{aligned}
& w_{+}^{2}=2 n e E, \\
& w_{-}^{2}=2(n+1) e E,
\end{aligned}
$$

and then one can write

$$
w^{2}=2 n e E,
$$

by taking into account that $n=0$ has degeneracy which is one half the degeneracy of $n>0$ (cf. also the $2 \mathrm{D}$ case in [39]).

As a consequence, the eigenvalues for $-D^{2}+\mu^{2}$ are

$$
\lambda^{2}=2 n e E+\mu_{k}^{2},
$$

where $\mu_{k}^{2}=2 \Lambda k^{2}+\mu^{2}$. Notice that the eigenvalues do not depend on $\omega$. An overall degeneracy factor $d$ must be determined. This can be obtained by comparing the behavior of the kernel $K(x)$ in $x=0$ to the universal coefficients provided by the heat kernel theorems. We have

$$
K(y)=\sum_{k} g(k) \exp \left(-\mu_{k}^{2} y\right)\left[d\left(2 \sum_{n=0}^{\infty} \exp (-2 n e E y)-1\right)\right] .
$$

The part in square bracket is the heat kernel for the $2 \mathrm{D}$ operator $-E^{2}$, and in the limit as $y \rightarrow 0$ it holds [ $\cdots] \sim 2 d(2 e E y)^{-1}$. As it must be equal to $2 S(4 \pi y)^{-1}$ (see [40], p. 368), where $S$ is the volume of the $2 \mathrm{D}$ space, we get $d=\frac{e E S}{2 \pi}$. We now define

$$
\zeta(s)=: \sum_{k} g(k) \zeta_{k}(s),
$$

with $^{4}$

$$
\begin{aligned}
\frac{1}{2} \zeta_{k}(s) & =\frac{d}{\Gamma(s)} \int_{0}^{\infty} d t t^{s-1} \sum_{n=0}^{\infty} \exp \left(-2 n e E t-\mu_{k}^{2} t\right)-\frac{1}{2} \frac{d}{\Gamma(s)} \int_{0}^{\infty} d t t^{s-1} \exp \left(-\mu_{k}^{2} t\right) \\
& =\frac{e E S}{2 \pi}\left[(2 e E)^{-s} \zeta_{H}\left(s, \frac{\mu_{k}^{2}}{2 e E}\right)-\frac{1}{\mu_{k}^{2 s}}\right]
\end{aligned}
$$

By putting $e E \mapsto i e E$ we finally obtain

$$
\begin{aligned}
\operatorname{Im} \frac{1}{2} \zeta_{k}^{\prime}(0) & =\frac{e E S}{2 \pi}\left[-\frac{1}{2} \log (2 e E)+\frac{\pi \mu_{k}^{2}}{4 e E}-\frac{1}{2} \log (2 \pi)+\operatorname{Re} \log \Gamma\left(-i \frac{\mu_{k}^{2}}{2 e E}\right)+\frac{1}{2} \log \left(\mu_{k}^{2}\right)\right] \\
& =\frac{e E S}{2 \pi}\left[-\frac{1}{2} \log \left(1-\exp \left(-\frac{\pi \mu_{k}^{2}}{e E}\right)\right)\right] .
\end{aligned}
$$

The volume factor appears here because of integration over the whole spacetime is included. This result agrees with the previous one.

\footnotetext{
${ }^{4}$ We introduce a factor $\frac{1}{2}$ in view of $(3.15)$.
} 


\section{The ultracold I case}

A second extremal limit of the Nariai background is given by the type I ultracold solution when $r_{-}=r_{+}=r_{c}$. The metric is [15]

$$
d s^{2}=-\chi^{2} d \psi^{2}+d \chi^{2}+\frac{1}{2 \Lambda}\left(d \theta^{2}+\sin ^{2}(\theta) d \phi^{2}\right),
$$

with $\chi \in(0, \infty)$ and $\psi \in \mathbb{R}$, and the electromagnetic field strength is $F=\sqrt{\Lambda} \chi d \chi \wedge d \psi$. The spacetime presents the structure of a $2 \mathrm{D}$ Rindler manifold times a two dimensional sphere (with a constant warping factor). One gets $\Gamma_{01}^{0}=\frac{1}{\chi}, \Gamma_{00}^{1}=\chi, \Gamma_{33}^{2}=-\sin (\theta) \cos (\theta), \Gamma_{23}^{3}=$ $\cot (\theta)$. We can choose $A_{0}=\frac{\sqrt{\Lambda}}{2} \chi^{2}$ and $A_{j}=0, j=1,2,3$ as potential. The situation is now a little bit more tricky, but we still are able to compare the transmission coefficient approach with the zeta function method. We expect to find out results which are analogous to some extent to the ones obtained in [45] for the case of a charged scalar field in a 2D Rindler spacetime with external electrostatic field.

\subsection{The transmission coefficient approach}

In [12] we have obtained that variable separation allows to obtain the following reduced Hamiltonian:

$$
h_{k}=\left[\begin{array}{cc}
-\frac{e \sqrt{\Lambda}}{2} \chi^{2}-\mu \chi & \chi \partial_{\chi}+\sqrt{2 \Lambda} k \chi \\
-\chi \partial_{\chi}+\sqrt{2 \Lambda} k \chi & -\frac{e \sqrt{\Lambda}}{2} \chi^{2}+\mu \chi
\end{array}\right]
$$

Again $\mu, e$ are the mass and the charge of the fermion. We also introduce $E:=\sqrt{\Lambda}$.

Using the coordinate $t=\chi^{2} / 2$ the Dirac equation takes the (Hamiltonian) form [12]

$$
\left[(e E t+\omega) I_{2}+\mu \sqrt{2 t} \sigma_{3}-i 2 t \sigma_{2} \partial_{t}-2 \sqrt{2 \Lambda} k \sigma_{1}\right] \zeta=0 .
$$

Rotating to $\xi$ by the $\sigma_{1}$ Pauli matrix as before: $\zeta=e^{-i \frac{\pi}{4} \sigma_{1}} \xi$, we get

$$
\begin{aligned}
& {\left[2 t \partial_{t}+i(e E t+\omega)\right] \xi_{1}-(\mu+i \sqrt{2 \Lambda} k) \sqrt{2 t} \xi_{2}=0,} \\
& {\left[2 t \partial_{t}-i(e E t+\omega)\right] \xi_{2}-(\mu-i \sqrt{2 \Lambda} k) \sqrt{2 t} \xi_{1}=0 .}
\end{aligned}
$$

This is equivalent to the system

$$
\begin{aligned}
(\mu+i \sqrt{2 \Lambda} k) \sqrt{2 t} \xi_{2} & =\left[2 t \partial_{t}+i(e E t+\omega)\right] \xi_{1} \\
0 & =t \xi_{1}^{\prime \prime}+\frac{1}{2} \xi_{1}^{\prime}-\frac{1}{4 t}\left[2 t \mu_{k}^{2}-(e E t+\omega)^{2}-i(e E t-\omega)\right] \xi_{1} .
\end{aligned}
$$

Set $\xi_{1}(t)=t^{-\frac{i}{2} \omega} e^{-\frac{i}{2} e E t} F(t)$. Then, $F$ must satisfy the confluent hypergeometric differential equation

$$
0=t F^{\prime \prime}+\left(\frac{1}{2}-i \omega-i e E t\right) F^{\prime}-\frac{\mu_{k}^{2}}{2} F
$$

which has general solution in terms of Kummer functions $\Phi(a ; c ; z)$ :

$$
F=\alpha \Phi\left(\frac{\mu_{k}^{2}}{2 i e E} ; \frac{1}{2}-i \omega ; i e E t\right)+\beta t^{\frac{1}{2}+i \omega} \Phi\left(\frac{1}{2}+i \omega+\frac{\mu_{k}^{2}}{2 i e E} ; \frac{3}{2}+i \omega ; i e E t\right) .
$$


Using the Kummer relation

$$
\Phi(a ; c ; z)=e^{z} \Phi(c-a ; c ;-z)
$$

we get

$\xi_{1}(t)=\alpha e^{-\frac{i}{2} e E t} t^{-\frac{i}{2} \omega} \Phi\left(\frac{\mu_{k}^{2}}{2 i e E} ; \frac{1}{2}-i \omega ; i e E t\right)+\beta e^{\frac{i}{2} e E t} t^{\frac{1}{2}+\frac{i}{2} \omega} \Phi\left(1-\frac{\mu_{k}^{2}}{2 i e E} ; \frac{3}{2}+i \omega ;-i e E t\right)$.

To compute $\xi_{2}$ we use (4.3) and

$$
\Phi^{\prime}(a ; c ; z)=\frac{a}{c} \Phi(a+1 ; c+1 ; z) .
$$

We get

$$
\begin{aligned}
\xi_{2}(t)= & \frac{i e E \alpha}{\mu+i \sqrt{2} E k} \frac{\mu_{k}^{2}}{i e E(1-2 i \omega)} e^{-\frac{i}{2} e E t} t^{-\frac{i}{2} \omega} \sqrt{2 t} \Phi\left(\frac{\mu_{k}^{2}}{2 i e E}+1 ; \frac{3}{2}-i \omega ; i e E t\right) \\
& -\frac{i e E \beta}{\mu+i \sqrt{2} E k} \frac{2 i e E-\mu_{k}^{2}}{i e E(3+2 i \omega)} e^{\frac{i}{2} e E t} t^{\frac{1}{2}+\frac{i}{2} \omega} \sqrt{2 t} \Phi\left(2-\frac{\mu_{k}^{2}}{2 i e E} ; \frac{5}{2}+i \omega ;-i e E t\right) \\
& +\frac{1+2 i(\omega+e E t)}{\sqrt{2 t}(\mu+i \sqrt{2} E k)} \beta e^{\frac{i}{2} e E t} t^{\frac{1}{2}+\frac{i}{2} \omega} \Phi\left(1-\frac{\mu_{k}^{2}}{2 i e E} ; \frac{3}{2}+i \omega ;-i e E t\right) .
\end{aligned}
$$

The asymptotic behaviors of these solutions are, for $t \approx 0$

$$
\begin{aligned}
& \xi_{1} \approx \alpha e^{-\frac{i}{2} e E t} t^{-\frac{i}{2} \omega}, \\
& \xi_{2} \approx \frac{\sqrt{2}}{\mu+i \sqrt{2} E k} \beta\left(i \omega+\frac{1}{2}\right) e^{\frac{i}{2} e E t} t^{\frac{i}{2} \omega},
\end{aligned}
$$

whereas for $t \approx+\infty$

$$
\begin{gathered}
\xi_{1} \approx e^{-\frac{i}{2} e E t} t^{-\frac{i}{2} \omega-\frac{\mu_{k}^{2}}{2 i e E}}\left[\alpha(-i e E)^{-\frac{\mu_{k}^{2}}{2 i e E}} \frac{\Gamma\left(\frac{1}{2}-i \omega\right)}{\Gamma\left(\frac{1}{2}-i \omega-\frac{\mu_{k}^{2}}{2 i e E}\right)}+\beta(-i e E)^{-\left(\frac{1}{2}+i \omega+\frac{\mu_{k}^{2}}{2 i e E}\right)} \frac{\Gamma\left(\frac{3}{2}+i \omega\right)}{\Gamma\left(1-\frac{\mu_{k}^{2}}{2 i e E}\right)}\right] \\
\xi_{2} \approx e^{\frac{i}{2} e E t} t^{\frac{i}{2} \omega+\frac{\mu_{k}^{2}}{2 i e E}} \frac{\sqrt{2}}{\mu+i \sqrt{2} E k}\left[\alpha(i e E)^{\frac{\mu_{k}^{2}}{2 i e E}+i \omega+\frac{1}{2}} \frac{\Gamma\left(\frac{1}{2}-i \omega\right)}{\Gamma\left(\frac{\mu_{k}^{2}}{2 i e E}\right)}+\beta(i e E)^{\frac{\mu_{k}^{2}}{2 i e E}} \frac{\Gamma\left(\frac{3}{2}+i \omega\right)}{\Gamma\left(\frac{1}{2}+i \omega+\frac{\mu_{k}^{2}}{2 i e E}\right)}\right] .
\end{gathered}
$$

It is useful to introduce a new variable $x:=\log (\chi)$. Using group velocity, (4.9) and (4.10) represent ingoing and outgoing particles respectively at $x \approx \infty$. Similarly, (4.7) and (4.8) are the outgoing and ingoing particle respectively at $x \approx-\infty$. In this situation, we can compute the transmission and reflection coefficients. Rewriting (4.7) and (4.8) as

$$
\xi_{1} \approx A e^{-i \phi(x)}, \quad \xi_{2} \approx B e^{i \phi(x)}
$$

and (4.9) and (4.10) as

$$
\xi_{1} \approx C e^{-i \phi(x)}, \quad \xi_{2} \approx D e^{i \phi(x)}
$$


with $\phi(x)=\left(\omega+\frac{\mu_{k}^{2}}{e E}\right) x+\frac{1}{2} e E e^{2 x}$, then we must put $C=0$ so that $R=A / B$ and $T=D / B$. We get

$$
\begin{aligned}
\left|R_{k}(\omega)\right|^{2} & =\frac{e^{-\pi \omega} \sinh \frac{\pi \mu_{k}^{2}}{2 e E}}{\cosh \left[\pi\left(\omega-\frac{\mu_{k}^{2}}{2 e E}\right)\right]}, \\
\left|T_{k}(\omega)\right|^{2} & =\frac{e^{-\frac{\pi \mu_{k}^{2}}{2 e E}} \cosh (\pi \omega)}{\cosh \left[\pi\left(\omega-\frac{\mu_{k}^{2}}{2 e E}\right)\right]} .
\end{aligned}
$$

As a check we note that $\left|T_{k}(\omega)\right|^{2}+\left|R_{k}(\omega)\right|^{2}=1$, and $\left|T_{k}(\omega)\right|^{2}$ gives the mean number of created pairs per unit time and unit volume.

A comparison with the result obtained in the WKB approximation in [12] is also in order. We recall that WKB gives for $\left|T_{k}\right|^{2}$ the same result as in the ultracold II case. Then, the dependence on $\omega$ is missing. We can relate this result with the exact one as follows: one considers $e E$ fixed and $\omega \rightarrow-\infty$. It is easily seen that the above exact result converges to the one of the WKB approximation in this limit.

We now calculate the imaginary part of the effective action. We write again

$$
\operatorname{Im} W:=\sum_{k} g(k) W_{k} .
$$

First of all, we note that there is an important difference with respect to the type II case. Here, the level-crossing region, i.e. the region where particle and antiparticle states overlap (cf. e.g. [8]), is no more the whole energy range but, assuming for definiteness $e E>0$, is determined by $\omega \leq 0$. As pair production is expected to happen only in the level-crossing region for the case $e E>0$, we must calculate

$$
W_{k}=-\frac{1}{2} \sum_{\omega} \log \left(1-\left|T_{k}(\omega)\right|^{2}\right)
$$

for $\omega \leq 0$. We have $\sum_{\omega} \mapsto \frac{\mathcal{T}}{2 \pi} \int d \omega$ (cf. [44, 45]), where $\mathcal{T}$ stays for a finite time interval, and it is easy to show that

$$
\log \left(1-\left|T_{i}\right|^{2}\right)=\log \left(1-\exp \left(-\frac{\pi \mu_{k}^{2}}{e E}\right)\right)-\log \left(1+\exp \left(2 \pi \omega-\frac{\pi \mu_{k}^{2}}{e E}\right)\right) .
$$

We have to evaluate the integral

$$
\int_{-\infty}^{0} d \omega \log \left(1+\exp \left(2 \pi \omega-\frac{\pi \mu_{k}^{2}}{e E}\right)\right)=-\frac{1}{2 \pi} \operatorname{Li}_{2}\left(-\exp \left(-\frac{\pi \mu_{k}^{2}}{e E}\right)\right),
$$

and then we get

$$
W_{k}=-\frac{1}{2} \frac{\mathcal{T}}{2 \pi}\left[\left(\int_{-\infty}^{0} d \omega\right) \log \left(1-\exp \left(-\frac{\pi \mu_{k}^{2}}{e E}\right)\right)+\frac{1}{2 \pi} \operatorname{Li}_{2}\left(-\exp \left(-\frac{\pi \mu_{k}^{2}}{e E}\right)\right)\right] .
$$

The factor $\frac{\mathcal{T}}{2 \pi}\left(\int_{-\infty}^{0} d \omega\right)$ amounts to a degeneracy factor which we can evaluate following [44]. As explained in the introduction the geometry of the ultracold I manifold is of the 
form of a Cartesian product $M^{1,1} \times S^{2}$, where the sphere could be considered an internal space. Thus, this situation can be treated in the same way as in [44] where a $1+1$ dimensional space time is taken into account. There, it is considered the Klein-Gordon equation for a complex scalar field in the presence of an $E$ field, but the same reasoning also works for a Dirac field. In this way we obtain a Schrödinger equation for a particle in an upside down oscillator potential and we can compute the degeneracy factor counting the number of modes whose turning points lie within $0<\psi<T$ and $0<\chi<L$, where $T$ and $L$ are the sizes of the space time box over which $E$ is nonvanishing. We put $T L=S$, and then we obtain for the degeneracy factor the value of $e E S / 2 \pi$, exactly the same as we will obtain by using the $\zeta$-function approach. We underline that (4.17) is in strict analogy with the results obtained in [45] for the scalar case in a $1+1$ Minkowski spacetime, and the terms appearing in (4.17) allow an analogous interpretation: the first contribution is leading and is a bulk one, proportional to the spacetime volume of the 2D Rindler part of the manifold, the latter one is a surface contribution. Cf. also [46].

\subsection{The $\zeta$-function approach}

The present case is also analyzed by means of $\zeta$-function techniques, which confirm the results obtained in the former approach.

For the Dirac operator on the $(\psi, \chi)$-part of the manifold one obtains

$$
E=\frac{1}{\chi} \tilde{\gamma}_{0}\left(\partial_{\psi}-i e E \frac{\chi^{2}}{2}\right)+\tilde{\gamma}_{1}\left(\partial_{\chi}+\frac{1}{2} \frac{1}{\chi}\right)
$$

A Liouville unitary transformation

$$
(S \Psi)(\psi, \chi):=\sqrt{\chi} \Psi(\psi, \chi)
$$

(i.e. $\left.\Psi(\psi, \chi)=\frac{1}{\sqrt{\chi}} \Phi(\psi, \chi)\right)$, leads to the unitarily related operator (still called $\left.\not E\right)$

$$
\not E=\frac{1}{\chi} \tilde{\gamma}_{0}\left(\partial_{\psi}-i e E \frac{\chi^{2}}{2}\right)+\tilde{\gamma}_{1} \partial_{\chi}
$$

and then

$$
E^{2}=\frac{1}{\chi^{2}}\left(\partial_{\psi}-i e E \frac{\chi^{2}}{2}\right)^{2}+\partial_{\chi}^{2}+\tilde{\gamma}_{0} \tilde{\gamma}_{1}\left(i e E \frac{1}{2}+\frac{1}{\chi^{2}} \partial_{\psi}\right)
$$

Substituting $t=\frac{\chi^{2}}{2}$, performing variable separation and keeping into account a unitary "rotation" transformation which is completely analogous to the one performed in the ultracold II case, one obtains for the eigenvalue equation of $-E^{2}$ the following couple of ordinary differential equations:

$$
t \partial_{t}^{2} \eta_{ \pm}(t)+\frac{1}{2} \partial_{t} \eta_{ \pm}(t)-\frac{1}{4 t}\left[(\omega+e E t)^{2} \pm(\omega-e E t)-2 t w^{2}\right] \eta_{ \pm}(t)=0 .
$$

Let us introduce $g_{ \pm}(t)$ through

$$
\eta_{ \pm}(t)=\exp \left( \pm \frac{1}{2} e E t\right) t^{ \pm \frac{1}{2} \omega} g_{ \pm}(t)
$$


then we obtain the following confluent hypergeometric equations:

$$
t \frac{d^{2} g_{ \pm}(t)}{d t^{2}}+\left[\frac{1}{2} \pm(\omega+e E t)\right] \frac{d g_{ \pm}(t)}{d t}+\frac{1}{2} w^{2} g_{ \pm}(t)=0 .
$$

We are looking for solutions $\left(\begin{array}{c}\eta_{+} \\ \eta_{-}\end{array}\right)$which belong to the Hilbert space $L^{2}\left[(0, \infty), \frac{d t}{t}\right]^{2}$ inherited from the original 4D space (the measure keeps into account the above Liouville transformation). We need to distinguish two regions for the energy $\omega$.

For $\omega<0$, we find

$$
g(t)_{-}=\Phi\left(\frac{-w^{2}}{2 e E} ; \frac{1}{2}-\omega ; e E t\right)
$$

with the quantization condition

$$
\frac{-w^{2}}{2 e E}=-n
$$

which gives

$$
w_{-}^{2}=2 e E n
$$

Moreover we find

$$
g(t)_{+}=t^{\frac{1}{2}-\omega} \exp (-e E t) \Phi\left(1-\frac{-w^{2}}{2 e E} ; \frac{3}{2}-\omega ; e E t\right),
$$

with the quantization condition

$$
1-\frac{w^{2}}{2 e E}=-n
$$

which gives

$$
w_{+}^{2}=2 e E(n+1) .
$$

Moreover, as in the ultracold II case, we can re-label the eigenvalues in such a way that the eigenvalues of $-\not^{2}+\mu^{2}$ in this region are

$$
\lambda^{2}=2 e E n+\mu_{k}^{2},
$$

where again $\mu_{k}^{2}=\mu^{2}+2 \Lambda k^{2}$. Also in this case the degeneracy of the $n=0$ case is one half the degeneracy of the $n>0$ cases, in full analogy with the ultracold II case. For the region $\omega>0$, solutions $\left(\begin{array}{c}\eta_{+} \\ \eta_{-}\end{array}\right) \in L^{2}\left[(0, \infty), \frac{d t}{t}\right]^{2}$ correspond to

$$
g_{-}(t)=t^{\frac{1}{2}+\omega} \Phi\left(\frac{1}{2}+\omega-\frac{w^{2}}{2 e E} ; \frac{3}{2}+\omega ; e E t\right),
$$

and to

$$
g_{+}(t)=\exp (-e E t) \Phi\left(\frac{1}{2}+\omega-\frac{w^{2}}{2 e E} ; \frac{1}{2}+\omega ; e E t\right),
$$

which are both associated with the quantization condition

$$
\frac{1}{2}+\omega-\frac{w^{2}}{2 e E}=-n
$$


The eigenvalues of $-\not D^{2}+\mu^{2}$ are for $\omega>0$

$$
\lambda^{2}=(2 n+1) e E+2 e E \omega+\mu_{k}^{2} .
$$

Note that the explicit dependence on $\omega$ appears only for the second region. For $\omega<0$, i.e. in the level-crossing region, we have independence on $\omega$ of the integrand. The degeneracy factor can be computed as for the ultracold II case giving the same factor. As to the term depending on $\omega$, with $\omega>0$, we recall that $\sum_{\omega} \mapsto \frac{\mathcal{T}}{2 \pi} \int d \omega$ holds. Then we obtain

$$
\frac{1}{2} \zeta_{k}(s)=\frac{e E S}{2 \pi}\left[(2 e E)^{-s} \zeta_{H}\left(\frac{\mu_{k}^{2}}{2 e E}, s\right)-\frac{1}{2} \frac{1}{\mu_{k}^{2 s}}\right]+\frac{\mathcal{T}}{2 \pi}(2 e E)^{-s} \frac{1}{s-1} \zeta_{H}\left(\frac{1}{2}\left(1+\frac{\mu_{k}^{2}}{e E}\right), s-1\right) .
$$

Note that the first term is the same as for the ultracold II case. Differentiating the second term with respect to $s$ in $s=0$ and going back to the Lorentzian signature we obtain the contributions

$$
\frac{\mathcal{T}}{2 \pi}\left[(\log (2 i e E)-1) \zeta_{H}\left(\frac{1}{2}\left[1+\frac{\mu_{k}^{2}}{i e E}\right],-1\right)+\frac{1}{2} \log \left(\mu_{k}^{2}\right)-\zeta_{H}^{\prime}\left(\frac{1}{2}\left[1+\frac{\mu_{k}^{2}}{i e E}\right],-1\right)\right] .
$$

To compute the imaginary part of this expression, we first note that

$$
\zeta_{H}(a,-1)=-\frac{1}{2} B_{2}(a)=-\frac{1}{2}\left(a^{2}-a+\frac{1}{6}\right),
$$

( $B_{2}(a)$ stays for the second Bernoulli polynomial) so that if $a$ is real

$$
\operatorname{Im} \zeta_{H}\left(\frac{1}{2}+i a,-1\right)=0 .
$$

Then, the first term gives the contribution

$$
\frac{1}{8 \pi}\left[\frac{1}{2}\left(\frac{\pi \mu_{k}^{2}}{e E}\right)^{2}+\frac{\pi^{2}}{6}\right] .
$$

To compute the second term, let us start from the obvious identity

$$
\zeta_{H}(a ; z)=\frac{1}{2^{z}} \zeta_{H}\left(\frac{a}{2} ; z\right)+\frac{1}{2^{z}} \zeta_{H}\left(\frac{a}{2}+\frac{1}{2} ; z\right) .
$$

Deriving in $z=-1$ and choosing $a=i x$ we get

$$
\zeta_{H}^{\prime}\left(i \frac{x}{2}+\frac{1}{2} ;-1\right)=\frac{1}{2} \zeta_{H}^{\prime}(i x ;-1)-\zeta_{H}^{\prime}\left(i \frac{x}{2} ;-1\right)+\frac{1}{2} \log 2 \zeta_{H}(i x ;-1) .
$$

Using $\operatorname{Im} \zeta_{H}(i x ;-1)=\frac{x}{2}$ and formula $(20)$ in $[42]$ we get

$$
\begin{aligned}
\operatorname{Im} \zeta_{H}^{\prime}\left(i \frac{x}{2}+\frac{1}{2} ;-1\right) & =\frac{1}{8 \pi}\left[-\frac{\pi^{2}}{6}-\frac{1}{2}(x \pi)^{2}-\sum_{n=1}^{\infty} \frac{e^{-2 n \pi x}}{n^{2}}+2 \sum_{n=1}^{\infty} \frac{e^{-n \pi x}}{n^{2}}\right] \\
& =\frac{1}{8 \pi}\left[-\frac{\pi^{2}}{6}-\frac{1}{2}(x \pi)^{2}-2 \sum_{n=1}^{\infty}(-1)^{n} \frac{e^{-n \pi x}}{n^{2}}\right] .
\end{aligned}
$$


Using the expression

$$
\sum_{n=1}^{\infty}(-1)^{n} \frac{e^{-n \pi x}}{n^{2}}=\operatorname{Li}_{2}\left(-e^{-\pi x}\right)
$$

and adding (4.39) and the derivative of the first term in (4.36), we finally get

$$
\frac{1}{2} \operatorname{Im} \zeta_{k}^{\prime}(0)=\frac{e E S}{2 \pi}\left[-\frac{1}{2} \log \left(1-\exp \left(-\frac{\pi \mu_{k}^{2}}{e E}\right)\right)\right]-\frac{\mathcal{T}}{2 \pi} \frac{1}{4 \pi} \operatorname{Li}_{2}\left(-\exp \left(-\frac{\pi \mu_{k}^{2}}{e E}\right)\right)
$$

This result coincides with (4.17). It is remarkable that the transmission coefficient approach involves only an integral over the level-crossing region, whereas the $\zeta$-function calculation requires a control over the whole spectrum, and is quite more tricky, but it provides also more information, being a priori the complete 1-loop effective action made available by the latter approach.

\subsection{Instability of the thermal state}

According to the general discussion of section 2, we get

$$
<N_{l}^{\text {out }}>_{\beta_{h}}=\frac{1}{1+\exp \left[2 \pi\left(\omega-\varphi^{+}\right)\right]}+\frac{e^{-\frac{\pi \mu_{k}^{2}}{2 e E}} \cosh (\pi \omega)}{\cosh \left[\pi\left(\omega-\frac{\mu_{k}^{2}}{2 e E}\right)\right]} \frac{1}{2}\left(\tanh \left[\pi\left(\omega-\varphi^{+}\right)\right]+\tanh \left[\pi\left(|\omega|+\varphi^{-}\right)\right]\right),
$$

where the latter term is the net effect associated with the pair production induced by the presence of an electrostatic field. Notice however that the potential $\varphi^{+}=\varphi^{-}$is ill-defined, being infinite unless a spatial cut-off is introduced at $\chi=\chi_{0}>0$.

\section{$5 \quad$ Nariai case}

We now consider the more general case, that is the electrically charged Nariai solution. The manifold is described by the metric [13-15]

$$
d s^{2}=\frac{1}{A}\left(-\sin ^{2}(\chi) d \psi^{2}+d \chi^{2}\right)+\frac{1}{B}\left(d \theta^{2}+\sin ^{2}(\theta) d \phi^{2}\right)
$$

with $\psi \in \mathbb{R}, \chi \in(0, \pi)$, and the constants $B=\frac{1}{2 Q^{2}}\left(1-\sqrt{1-12 \frac{Q^{2}}{L^{2}}}\right), A=\frac{6}{L^{2}}-B$ are such that $\frac{A}{B}<1$, and $L^{2}:=\frac{3}{\Lambda}$. The black hole horizon occurs at $\chi=\pi$. This manifold differs from the ultracold cases because it has finite spatial section. In the Euclidean version, it corresponds to two spheres characterized by different radii. One finds the following non-vanishing Christoffel symbols $\Gamma_{01}^{0}=\cot (\chi), \Gamma_{00}^{1}=\sin (\chi) \cos (\chi), \Gamma_{33}^{2}=$ $-\sin (\theta) \cos (\theta), \Gamma_{23}^{3}=\cot (\theta)$. For the gauge potential we can choose $A_{i}=-Q \frac{B}{A} \cos (\chi) \delta_{i}^{0}$.

As the situation is more difficult, in this section we will provide a more detailed exposition. 


\subsection{The transmission coefficient approach}

By variable separation, as performed in [12], we obtain the following reduced Hamiltonian (where $\mu, e$ are the fermion mass and charge, as before):

$$
h_{k}=\left[\begin{array}{cc}
e Q \frac{B}{A} \cos (\chi)-\frac{\mu}{\sqrt{A}} \sin (\chi) & \sin (\chi) \partial_{\chi}+\sqrt{\frac{B}{A}} \sin (\chi) k \\
-\sin (\chi) \partial_{\chi}+\sqrt{\frac{B}{A}} \sin (\chi) k & e Q \frac{B}{A} \cos (\chi)+\frac{\mu}{\sqrt{A}} \sin (\chi)
\end{array}\right] .
$$

We introduce, with the aim of simplifying the notation, the following definition:

$$
E:=Q \frac{B}{A},
$$

which corresponds to $\frac{1}{A}$ times the maximum value for the intensity of the electrostatic field. Moreover, we assume for definiteness $e E>0$, and also we adopt the following redefinitions:

$$
\begin{aligned}
& \frac{1}{\sqrt{A}} \mu \mapsto \mu, \\
& \sqrt{\frac{B}{A}} k \mapsto k .
\end{aligned}
$$

Then the Dirac equation in the Hamiltonian form is

$$
\left(\begin{array}{cc}
e E \cos \chi-\mu \sin \chi & \sin \chi \partial_{\chi}+k \sin \chi \\
-\sin \chi \partial_{\chi}+k \sin \chi & e E \cos \chi+\mu \sin \chi
\end{array}\right)\left(\begin{array}{l}
\psi_{1} \\
\psi_{2}
\end{array}\right)=\omega\left(\begin{array}{l}
\psi_{1} \\
\psi_{2}
\end{array}\right) .
$$

Using the coordinate $t=-\cos \chi$ we can write it in the form

$$
\left[(e E t+\omega) \mathbb{I}_{2}+\mu \sqrt{1-t^{2}} \sigma_{3}-i\left(1-t^{2}\right) \sigma_{2} \partial_{t}-k \sqrt{1-t^{2}} \sigma_{1}\right] \Psi=0,
$$

where $\Psi=\left(\begin{array}{l}\psi_{1} \\ \psi_{2}\end{array}\right)$. Let us take the unitary transformation $\Psi=e^{-i \frac{\pi}{4} \sigma_{1}} \xi$; then

$$
\begin{aligned}
& {\left[\left(1-t^{2}\right) \partial_{t}+i(e E t+\omega)\right] \xi_{1}-(\mu+i k) \sqrt{1-t^{2}} \xi_{2}=0} \\
& {\left[\left(1-t^{2}\right) \partial_{t}-i(e E t+\omega)\right] \xi_{2}-(\mu-i k) \sqrt{1-t^{2}} \xi_{1}=0}
\end{aligned}
$$

From (5.6) we find

$$
(\mu+i k) \xi_{2}=\sqrt{1-t^{2}} \xi_{1}^{\prime}+i \frac{e E t+\omega}{\sqrt{1-t^{2}}} \xi_{1},
$$

which inserted into (5.7) gives

$$
\left(1-t^{2}\right) \xi_{1}^{\prime \prime}-t \xi_{1}^{\prime}-\left(\mu^{2}+k^{2}-i e E\right) \xi_{1}+\frac{1}{1-t^{2}}\left[(e E t+\omega)^{2}+i(e E t+\omega) t\right] \xi_{1}=0 .
$$

Looking at the behavior of this equation at the singular points $t= \pm 1$ we see that it is convenient to define a function $\zeta$ such that

$$
\begin{aligned}
\xi_{1}(t) & =(1-t)^{\alpha}(1+t)^{\beta} \zeta((1+t) / 2), \\
\alpha & \in\left\{\frac{1}{2}(1-i e E-i \omega), \frac{i}{2}(e E+\omega)\right\}, \quad \beta \in\left\{\frac{1}{2}(1-i e E+i \omega), \frac{i}{2}(e E-\omega)\right\} .
\end{aligned}
$$


Setting $z=(1+t) / 2$ and choosing $\alpha=\frac{i}{2}(e E+\omega)$ and $\beta=\frac{i}{2}(e E-\omega)$, we find

$$
z(1-z) \zeta^{\prime \prime}+\left[2 \beta+\frac{1}{2}-z(1+2 i e E)\right] \zeta^{\prime}-\left[\mu^{2}+k^{2}\right] \zeta=0
$$

which is an hypergeometric differential equation. Note that the solution regular in $z=0$ of this equation provides a solution of (5.9) regular in $t=-1$. Moreover, (5.9) is invariant under the combination of complex conjugation with the transformation $(t, E) \rightarrow(-t,-E)$. Thus, we are able to get the solution regular in $t=1$ applying this transformation to the solution regular in $t=-1$. This gives

$$
\begin{aligned}
\xi_{1}(t)= & c_{1}\left(\frac{1-t}{2}\right)^{i \frac{e E+\omega}{2}}\left(\frac{1+t}{2}\right)^{i \frac{e E-\omega}{2}}{ }_{2} F_{1}\left(i e E+i \sqrt{\Delta}, i e E-i \sqrt{\Delta} ; \frac{1}{2}+i(e E-\omega) ; \frac{1+t}{2}\right) \\
& +c_{2}\left(\frac{1-t}{2}\right)^{i \frac{e E+\omega}{2}}\left(\frac{1+t}{2}\right)^{i \frac{e E-\omega}{2}}{ }_{2} F_{1}\left(i e E+i \sqrt{\Delta}, i e E-i \sqrt{\Delta} ; \frac{1}{2}+i(e E+\omega) ; \frac{1-t}{2}\right)
\end{aligned}
$$

where $\Delta:=\mu^{2}+k^{2}+e^{2} E^{2}$, and ${ }_{2} F_{1}$ is the well-known Gauss hypergeometric function.

Using (5.8) and the relations

$$
\begin{aligned}
& -\sqrt{1-t^{2}} \frac{d}{d t}\left[\left(\frac{1-t}{2}\right)^{i \frac{e E+\omega}{2}}\left(\frac{1+t}{2}\right)^{i \frac{e E-\omega}{2}}\right]-i \frac{e E t+\omega}{\sqrt{1-t^{2}}}\left[\left(\frac{1-t}{2}\right)^{i \frac{e E+\omega}{2}}\left(\frac{1+t}{2}\right)^{i \frac{e E-\omega}{2}}\right]=0 \\
& { }_{2} F_{1}^{\prime}(a, b ; c ; z)=\frac{a b}{c}{ }_{2} F_{1}(a+1, b+1 ; c+1 ; z),
\end{aligned}
$$

we get

$$
\begin{aligned}
\xi_{2}(t)= & c_{1} \frac{2(\mu-i k)}{1+2 i(e E-\omega)}\left(\frac{1-t}{2}\right)^{i \frac{e E+\omega}{2}+\frac{1}{2}}\left(\frac{1+t}{2}\right)^{i \frac{e E-\omega}{2}+\frac{1}{2}} \\
& { }_{2} F_{1}\left(i e E+i \sqrt{\Delta}+1, i e E-i \sqrt{\Delta}+1 ; \frac{3}{2}+i(e E-\omega) ; \frac{1+t}{2}\right) \\
& -c_{2} \frac{2(\mu-i k)}{1+2 i(e E+\omega)}\left(\frac{1-t}{2}\right)^{i \frac{e E+\omega}{2}+\frac{1}{2}}\left(\frac{1+t}{2}\right)^{i \frac{e E-\omega}{2}+\frac{1}{2}} \\
& { }_{2} F_{1}\left(i e E+i \sqrt{\Delta}+1, i e E-i \sqrt{\Delta}+1 ; \frac{3}{2}+i(e E+\omega) ; \frac{1-t}{2}\right) .
\end{aligned}
$$

As in the previous cases, we can look at the asymptotic behaviors at infinities, in the coordinate $x=\log \tan \frac{\chi}{2}$. For $x \rightarrow-\infty$ we get

$$
\begin{aligned}
& \xi_{1}(x) \approx\left[c_{1}+c_{2} \frac{\Gamma\left(\frac{1}{2}+i(e E+\omega)\right) \Gamma\left(\frac{1}{2}-i(e E-\omega)\right)}{\Gamma\left(\frac{1}{2}+i \omega-i \sqrt{\Delta}\right) \Gamma\left(\frac{1}{2}+i \omega+i \sqrt{\Delta}\right)}\right] e^{i(e E-\omega) x}+e^{-i(e E-\omega) x} O\left(e^{x}\right), \quad \text { (5.15) } \\
& \xi_{2}(x) \approx-c_{2} \frac{2(\mu-i k)}{1+2 i(e E+\omega)} \frac{\Gamma\left(\frac{3}{2}+i(e E+\omega)\right) \Gamma\left(\frac{1}{2}+i(e E-\omega)\right)}{\Gamma(1+i e E-i \sqrt{\Delta}) \Gamma(1+i e E+i \sqrt{\Delta})} e^{-i(e E-\omega) x}+e^{i(e E-\omega) x} O\left(e^{x}\right) .
\end{aligned}
$$


For $x \rightarrow+\infty:$

$$
\begin{aligned}
& \xi_{1}(x) \approx\left[c_{2}+c_{1} \frac{\Gamma\left(\frac{1}{2}+i(e E-\omega)\right) \Gamma\left(\frac{1}{2}-i(e E+\omega)\right)}{\Gamma\left(\frac{1}{2}-i \omega-i \sqrt{\Delta}\right) \Gamma\left(\frac{1}{2}-i \omega+i \sqrt{\Delta}\right)}\right] e^{-i(e E+\omega) x}+e^{i(e E+\omega) x} O\left(e^{-x}\right), \quad \text { (5.17) } \\
& \xi_{2}(x) \approx c_{1} \frac{2(\mu-i k)}{1+2 i(e E-\omega)} \frac{\Gamma\left(\frac{3}{2}+i(e E-\omega)\right) \Gamma\left(\frac{1}{2}+i(e E+\omega)\right)}{\Gamma(1+i e E-i \sqrt{\Delta}) \Gamma(1+i e E+i \sqrt{\Delta})} e^{i(e E+\omega) x}+e^{-i(e E+\omega) x} O\left(e^{-x}\right) .
\end{aligned}
$$

To compute the transmission and reflection coefficient let us write the asymptotic expressions for $\xi$ as

$$
\begin{aligned}
& \xi_{1}(x)^{-} \approx\left[c_{1}+c_{2} \alpha_{0}\right] e^{i(e E-\omega) x}+e^{-i(e E-\omega) x} O\left(e^{x}\right), \\
& \xi_{2}(x)^{-} \approx-c_{2} \beta_{0} e^{-i(e E-\omega) x}+e^{i(e E-\omega) x} O\left(e^{x}\right),
\end{aligned}
$$

for $x \rightarrow-\infty$, and for $x \rightarrow \infty$

$$
\begin{aligned}
& \xi_{1}(x)^{+} \approx\left[c_{2}+c_{1} \alpha_{1}\right] e^{-i(e E+\omega) x}+e^{i(e E+\omega) x} O\left(e^{-x}\right) \\
& \xi_{2}(x)^{+} \approx c_{1} \beta_{1} e^{i(e E+\omega) x}+e^{-i(e E+\omega) x} O\left(e^{-x}\right)
\end{aligned}
$$

By taking into account correctly group velocity and imposing that there is only incoming wave at $x=-\infty$, one finds

$$
\begin{aligned}
\left|T_{k}(\omega)\right|^{2} & =\frac{\cosh [\pi(e E-\omega)] \cosh [\pi(e E+\omega)]}{\cosh [\pi(\sqrt{\Delta}-\omega)] \cosh [\pi(\sqrt{\Delta}+\omega)]} \\
& =\frac{\cosh [2 \pi e E]+\cosh [2 \pi \omega]}{\cosh [2 \pi \sqrt{\Delta}]+\cosh [2 \pi \omega]}
\end{aligned}
$$

which has the expected property to satisfy $|T|^{2}<1$ and gives the mean number of created pairs for unit time and unit volume. Moreover, we get

$$
\left|R_{k}(\omega)\right|^{2}=\frac{\sinh [\pi(\sqrt{\Delta}-e E)] \sinh [\pi(\sqrt{\Delta}+e E)]}{\cosh [\pi(\sqrt{\Delta}-\omega)] \cosh [\pi(\sqrt{\Delta}+\omega)]},
$$

and the property $\left|T_{k}(\omega)\right|^{2}+\left|R_{k}(\omega)\right|^{2}=1$ is verified. We recall that one has to reinstate in the above formulas for $\left|T_{k}\right|^{2}$ the original values for $\mu$ and $k$ (cf. eq. (5.4)). Then $\Delta=$ $\frac{\mu^{2}}{A}+\frac{B}{A} k^{2}+(e E)^{2}$.

Comparing with [12], the limit as $e E \rightarrow \infty$ leads to the WKB approximation (and to the limit $\left.\left|T_{k}(\omega)\right|^{2} \rightarrow 1^{-}\right)$. This is the actual approximation where the WKB approximation works well. We can notice that the above limit actually means $Q \frac{B}{A} \rightarrow \infty$. Being

$$
\frac{B}{A}=\frac{1}{\sqrt{1-4 \Lambda Q^{2}}}
$$

one can obtain the above limit as $Q^{2} \rightarrow\left(\frac{1}{4 \Lambda}\right)^{-}$. 
In order to determine the imaginary part of the effective action, we refer again to (4.13). We do not perform the sum over $k$, and then we calculate

$$
W_{k}=-\frac{1}{2} \sum_{\omega} \log \left(1-\left|T_{k}(\omega)\right|^{2}\right)
$$

We sum only over the level-crossing region, because only there particle creation is expected to be present, and then only there an instability for the vacuum should occur. This region is $-e E \leq \omega \leq e E$ so that we have to perform the following integral:

$$
I:=\int_{-e E}^{e E} d \omega \log \left(1-\frac{\cosh [2 \pi e E]+\cosh [2 \pi \omega]}{\cosh [2 \pi \sqrt{\Delta}]+\cosh [2 \pi \omega]}\right),
$$

where the dependence on $k$ is implicit in $\Delta$; the integral can be rewritten as follows:

$$
I=2 e E \log (\cosh [2 \pi \sqrt{\Delta}]-\cosh [2 \pi e E])-I I,
$$

where

$$
I I:=\int_{-e E}^{e E} d \omega \log (\cosh [2 \pi \sqrt{\Delta}]+\cosh [2 \pi \omega])=\frac{1}{2 \pi} \int_{-2 \pi e E}^{2 \pi e E} d y \log (p+\cosh [y]),
$$

with $p:=\cosh [2 \pi \sqrt{\Delta}]$. By taking into account that $p+\cosh [y]=$ $\frac{1}{2}(2 p+\exp (y)+\exp (-y))=\frac{1}{2} \exp (y)(\exp (-2 y)+2 p \exp (-y)+1)=$ $\frac{1}{2} \exp (y)\left(\exp (-y)+p-\sqrt{p^{2}-1}\right)\left(\exp (-y)+p+\sqrt{p^{2}-1}\right)$ and that $p-\sqrt{p^{2}-1}=$ $\exp (-2 \pi \sqrt{\Delta})$ and $p+\sqrt{p^{2}-1}=\exp (2 \pi \sqrt{\Delta})$, one finds

$I I=\frac{1}{2 \pi} \int_{-2 \pi e E}^{2 \pi e E} d y[y-\log 2+\log (\exp (-y) \exp (-2 \pi \sqrt{\Delta})+1)+\log (\exp (-y) \exp (2 \pi \sqrt{\Delta})+1)]$.

The following result is useful:

$$
\begin{aligned}
\int d y \log (\exp (-y+\delta)+1)= & \frac{1}{2} y^{2}+y \log (\exp (-y+\delta)+1)-y \log (\exp (y-\delta)+1) \\
& -\operatorname{Li}_{2}(-\exp (y-\delta)) \\
= & -\frac{1}{2} y^{2}+y \delta-\operatorname{Li}_{2}(-\exp (y-\delta)) .
\end{aligned}
$$

As a consequence, with simple manipulations, we get

$$
\begin{aligned}
I I= & \frac{1}{2 \pi}\left[-2(2 \pi e E) \log 2+\operatorname{Li}_{2}(-\exp [-2 \pi(\sqrt{\Delta}+e E)])-\operatorname{Li}_{2}(-\exp [2 \pi(\sqrt{\Delta}+e E)])\right. \\
& \left.+\operatorname{Li}_{2}(-\exp [2 \pi(\sqrt{\Delta}-e E)])-\mathrm{Li}_{2}(-\exp [-2 \pi(\sqrt{\Delta}-e E)])\right],
\end{aligned}
$$

and then, by taking into account that $\sum_{\omega} \mapsto \frac{\mathcal{I}}{2 \pi} \int d \omega$, we get

$$
\begin{aligned}
W_{k}= & -\frac{\mathcal{T}}{2 \pi} e E \log (2 \cosh [2 \pi \sqrt{\Delta}]-2 \cosh [2 \pi e E])-\frac{\mathcal{T}}{2 \pi} \frac{1}{4 \pi}\left[-\operatorname{Li}_{2}(-\exp [-2 \pi(\sqrt{\Delta}+e E)])\right. \\
& +\operatorname{Li}_{2}(-\exp [2 \pi(\sqrt{\Delta}+e E)])-\operatorname{Li}_{2}(-\exp [2 \pi(\sqrt{\Delta}-e E)]) \\
& \left.+\operatorname{Li}_{2}(-\exp [-2 \pi(\sqrt{\Delta}-e E)])\right] .
\end{aligned}
$$


In the Nariai case, it is not so straightforward to distinguish between bulk and surface parts of the given $W_{k}$. By keeping into account that $E \propto \frac{1}{A}$ and that the volume of the (Euclidean) 2D $(\psi, \chi)$-part of the metric is $\frac{4 \pi}{A}$, one realizes that the first above contribution is leading and is a bulk one and the latter is again a surface contribution.

\subsection{The $\zeta$-function approach}

This case requires more efforts in order to be tackled with $\zeta$-function techniques, in particular it needs techniques which have been developed only recently [20], and we sketch herein a more heuristic approach which still leads to the desired results.

As to the operator $\not$ on the $(\psi, \chi)$-part of the manifold, one obtains

$$
\text { E }=\frac{\sqrt{A}}{\sin \chi} \tilde{\gamma}_{0}\left(\partial_{\psi}+i e E \cos \chi\right)+\sqrt{A} \tilde{\gamma}_{1}\left(\partial_{\chi}+\frac{1}{2} \cot \chi\right) .
$$

After a Liouville unitary transformation $\Psi(\psi, \chi)=\frac{1}{\sqrt{\sin \chi}} \phi(\psi, \chi)$, we get the simplified form (again called $\not$ )

$$
\not E=\frac{\sqrt{A}}{\sin \chi} \tilde{\gamma}_{0}\left(\partial_{\psi}+i e E \cos \chi\right)+\sqrt{A} \tilde{\gamma}_{1} \partial_{\chi}
$$

moreover,

$$
E^{2}=\frac{A}{\sin ^{2} \chi}\left(\partial_{\psi}+i e E \cos \chi\right)^{2}+A \partial_{\chi}^{2}+A \tilde{\gamma}_{0} \tilde{\gamma}_{1}\left(\frac{\cos \chi}{\sin ^{2} \chi}\left(\partial_{\psi}+i e E \cos \chi\right)+i e E\right) .
$$

Substituting $t=-\cos \chi$ one obtains

$$
E^{2}=\frac{A}{1-t^{2}}\left(\partial_{\psi}-i e E t\right)^{2}+A\left(1-t^{2}\right) \partial_{t}^{2}-A t \partial_{t}+A \tilde{\gamma}_{0} \tilde{\gamma}_{1}\left(-\frac{t}{1-t^{2}}\left(\partial_{\psi}-i e E t\right)+i e E\right)
$$

After variable separation, a reduction and a rotation as in the previous cases, we obtain for the eigenvalue problem of $-E^{2}$ the following couple of differential equations:

$$
\left(1-t^{2}\right) \partial_{t}^{2} \eta_{ \pm}-t \partial_{t} \eta_{ \pm} \pm e E \eta_{ \pm}+\frac{1}{1-t^{2}}\left[-(\omega+e E t)^{2} \pm t(\omega+e E t)\right] \eta_{ \pm}+\frac{w^{2}}{A} \eta_{ \pm}=0
$$

We choose

$$
\begin{aligned}
& \eta_{+}(t)=(1-t)^{\frac{e E+\omega}{2}}(1+t)^{\frac{e E-\omega}{2}} g_{+}(t) \\
& \eta_{-}(t)=(1-t)^{\frac{-e E-\omega}{2}}(1+t)^{\frac{-e E+\omega}{2}} g_{-}(t)
\end{aligned}
$$

Then by choosing $t=2 z-1$ we obtain the following couple of hypergeometric equations:

$$
z(1-z) \frac{d^{2} g_{+}(z)}{d z^{2}}+\left(e E-\omega+\frac{1}{2}-(2 e E+1) z\right) \frac{d g_{+}(z)}{d z}+\frac{w^{2}}{A} g_{+}(z)=0
$$

with $a_{+}=e E+\sqrt{\frac{w^{2}}{A}+(e E)^{2}}, b_{+}=e E-\sqrt{\frac{w^{2}}{A}+(e E)^{2}}, c_{+}=e E-\omega+\frac{1}{2}$, and

$$
z(1-z) \frac{d^{2} g_{-}(z)}{d z^{2}}+\left(-e E+\omega+\frac{1}{2}+(2 e E-1) z\right) \frac{d g_{-}(z)}{d z}+\frac{w^{2}}{A} g_{-}(z)=0
$$


with $a_{-}=-e E+\sqrt{\frac{w^{2}}{A}+(e E)^{2}}, b_{-}=-e E-\sqrt{\frac{w^{2}}{A}+(e E)^{2}}, c_{-}=-e E+\omega+\frac{1}{2}$. We are looking for solutions $\left(\begin{array}{c}\eta_{+} \\ \eta_{-}\end{array}\right) \in L^{2}\left[(0,1), \frac{d z}{z(1-z)}\right]^{2}$. It is not difficult to realize that this condition depends on $\omega$, and three regions can be identified. One can show that

$$
g_{+}(z)={ }_{2} F_{1}\left(a_{+}, b_{+} ; c_{+} ; z\right)
$$

with the quantization condition

$$
b_{+}=-n,
$$

which leads to

$$
w_{+}^{2}=A(e E+n)^{2}-A(e E)^{2},
$$

together with

$$
g_{-}(z)=z^{1-c_{-}}(1-z)^{c_{--}\left(a_{-}+b_{-}\right)}{ }_{2} F_{1}\left(1-a_{-}, 1-b_{-} ; 2-c_{-} ; z\right)
$$

with the quantization condition

$$
1-a_{-}=-n
$$

which leads to

$$
w_{-}^{2}=A(e E+n+1)^{2}-A(e E)^{2},
$$

correspond to solutions of $(5.41),(5.42)$ respectively which allow to get $\left(\begin{array}{c}\eta_{+} \\ \eta_{-}\end{array}\right) \in$ $L^{2}\left[(0,1), \frac{d z}{z(1-z)}\right]^{2}$ in the interval $-e E<\omega<e E$. We can re-label the eigenvalue and obtain for $-D^{2}+\mu^{2}$

$$
\lambda^{2}=A(e E+n)^{2}-A(e E)^{2}+\mu^{2}+B k^{2}
$$

Also in this case, one has to take into account that degeneracy for $n=0$ is one half the degeneracy for $n>0$.

In the region $\omega>e E$ one can show that the couple

$$
g_{+}(z)=z^{1-c_{+}}{ }_{2} F_{1}\left(a_{+}-c_{+}+1, b_{+}-c_{+}+1 ; 2-c_{+} ; z\right)
$$

and

$$
g_{-}(z)=(1-z)^{c_{-}-\left(a_{-}+b_{-}\right)}{ }_{2} F_{1}\left(c_{-}-a_{-}, c_{-}-b_{-} ; c_{-} ; z\right)
$$

under the quantization conditions $b_{+}-c_{+}+1=-n$ and $c_{-}-a_{-}=-n$, which both lead to

$$
w^{2}=A\left(\omega+\frac{1}{2}+n\right)^{2}-A(e E)^{2}
$$

provides a solution which is in $L^{2}\left[(0,1), \frac{d z}{z(1-z)}\right]^{2}$. Then the eigenvalue of $-D^{2}+\mu^{2}$ becomes

$$
\lambda^{2}=A\left(\omega+n+\frac{1}{2}\right)^{2}-A(e E)^{2}+\mu^{2}+B k^{2}
$$


There is still the region $\omega<-e E$ to be explored, where

$$
g_{+}(z)=(1-z)^{c_{+}-\left(a_{+}+b_{+}\right)}{ }_{2} F_{1}\left(c_{+}-b_{+}, c_{+}-a_{+} ; c_{+}-\left(a_{+}+b_{+}\right)+1 ; 1-z\right),
$$

and

$$
g_{-}(z)=z^{1-c_{-}}{ }_{2} F_{1}\left(1+b_{-}-c_{-}, 1+a_{-}-c_{-} ; a_{-}+b_{-}+1-c_{-} ; 1-z\right)
$$

with the quantization conditions $c_{+}-a_{+}=-n$ and $1+b_{-}-c_{-}=-n$, which both correspond to

$$
w^{2}=A\left(-\omega+\frac{1}{2}+n\right)^{2}-A(e E)^{2},
$$

satisfy the property to be eigenfunctions of the operator $-E^{2}$. The eigenvalues of $-D^{2}+\mu^{2}$ are

$$
\lambda^{2}=A\left(-\omega+n+\frac{1}{2}\right)^{2}-A(e E)^{2}+\mu^{2}+B k^{2} .
$$

Note that, for $|\omega|>e E$ the eigenvalues of $-D^{2}+\mu^{2}$ can be written as follows:

$$
\lambda^{2}=A\left(|\omega|+n+\frac{1}{2}\right)^{2}+\mu^{2}+B k^{2}-A(e E)^{2},
$$

and then the integration for $|\omega|>e E$ is symmetric.

For the heat kernel we get

$$
K(s)=\sum_{k} g(k) K_{k}(s)
$$

with

$$
\begin{aligned}
K_{k}(s)= & 2 \frac{\mathcal{T}}{2 \pi}\left\{2 \int_{e E}^{\infty} d \omega \sum_{n=0}^{\infty} \exp \left[-A\left(\left(\omega+\frac{1}{2}+n\right)^{2}+\frac{\mu_{k}^{2}}{A}-(e E)^{2}\right) s\right]\right. \\
& \left.+2 e E \sum_{n=0}^{\infty} \exp \left[-A\left((e E+n)^{2}+\frac{\mu_{k}^{2}}{A}-(e E)^{2}\right) s\right]-e E \exp \left(-\mu_{k}^{2} s\right)\right\},
\end{aligned}
$$

where $\mu_{k}^{2}=\mu^{2}+B k^{2}$. Correspondingly, we obtain

$$
\begin{aligned}
\frac{1}{2} \zeta_{k}(s)=\frac{\mathcal{T}}{2 \pi} & {\left[2 \int_{e E}^{\infty} \sum_{n} \frac{d \omega}{A^{s}\left[\left(n+\frac{1}{2}+\omega\right)^{2}+\frac{\mu_{k}^{2}}{A}-(e E)^{2}\right]^{s}}+\right.} \\
& \left.+(2 e E)\left(\sum_{n} \frac{1}{A^{s}\left[(n+e E)^{2}+\frac{\mu_{k}^{2}}{A}-(e E)^{2}\right]^{s}}-\frac{1}{2} \frac{1}{\mu_{k}^{2 s}}\right)\right] .
\end{aligned}
$$

It is convenient to introduce the functions

$$
\sigma_{k}(s ; z):=\sum_{n} \frac{1}{A^{s}\left[\left(n+\frac{1}{2}+e E+z\right)^{2}+\frac{\mu_{k}^{2}}{A}-(e E)^{2}\right]^{s}},
$$


so that

$$
\frac{1}{2} \zeta_{k}(s)=\frac{\mathcal{T}}{2 \pi}\left[2 e E\left(\sigma_{k}\left(s ;-\frac{1}{2}\right)-\frac{1}{2} \frac{1}{\mu_{k}^{2 s}}\right)+2 \int_{0}^{\infty} \sigma_{k}(s ; z) d z\right] .
$$

It is also useful to define $\alpha=\frac{1}{2}+e E$ and $\beta^{2}=\frac{\mu_{k}^{2}}{A}-(e E)^{2}$. Using the Abel-Plana formula ${ }^{5}$ we get

$$
\begin{aligned}
\sigma_{k}(s ; z)= & \frac{1}{2} \frac{1}{A^{s}\left[(\alpha+z)^{2}+\beta^{2}\right]^{s}}+\int_{0}^{\infty} \frac{d x}{A^{s}\left[(x+\alpha+z)^{2}+\beta^{2}\right]^{s}} \\
& +i \int_{0}^{\infty} d x\left\{\frac{1}{A^{s}\left[(i x+\alpha+z)^{2}+\beta^{2}\right]^{s}}-\frac{1}{A^{s}\left[(-i x+\alpha+z)^{2}+\beta^{2}\right]^{s}}\right\} \frac{1}{e^{2 \pi x}-1} .
\end{aligned}
$$

To compute the effective action, we need to compute the derivative of $\sigma_{k}(s ; z)$ with respect to $s$, in $s=0$. For the last term of (5.64) we get

$$
-i \int_{0}^{\infty} d x\left\{\ln \left[(i x+\alpha+z)^{2}+\beta^{2}\right]-\ln \left[(-i x+\alpha+z)^{2}+\beta^{2}\right]\right\} \frac{1}{e^{2 \pi x}-1} .
$$

Note that this coincides with

$$
\begin{aligned}
\left.i \frac{d}{d s}\right|_{s=0} \int_{0}^{\infty} d x & \frac{1}{A^{\gamma s}}\left\{\frac{1}{(i x+\alpha+z+i \beta)^{s}}-\frac{1}{(-i x+\alpha+z+i \beta)^{s}}\right. \\
& \left.+\frac{1}{(i x+\alpha+z-i \beta)^{s}}-\frac{1}{(-i x+\alpha+z-i \beta)^{s}}\right\} \frac{1}{e^{2 \pi x}-1},
\end{aligned}
$$

where $\gamma$ is an arbitrary constant. The result does not depend on $\gamma$ and we could choose it equal to 0 . However, it is convenient to choose $\gamma=\frac{1}{2}$. Applying Plana's formula to this integrals we see that

$$
\left.\frac{\partial}{\partial s}\right|_{s=0} \sigma_{k}(s ; z)=\left.\frac{\partial}{\partial s}\right|_{s=0} \tilde{\sigma}_{k}(s ; z)
$$

where

$$
\begin{aligned}
\tilde{\sigma}_{k}(s ; z)= & \frac{1}{2} \frac{1}{A^{s}\left[(\alpha+z)^{2}+\beta^{2}\right]^{s}}+\int_{0}^{\infty} \frac{d x}{A^{s}\left[(x+\alpha+z)^{2}+\beta^{2}\right]^{s}} \\
& +\frac{1}{A^{s / 2}} \sum_{n=0}^{\infty}\left\{\frac{1}{(n+\alpha+z+i \beta)^{s}}+\frac{1}{(n+\alpha+z-i \beta)^{s}}\right\} \\
& -\frac{1}{2} \frac{1}{A^{s / 2}}\left\{\frac{1}{(\alpha+z+i \beta)^{s}}+\frac{1}{(\alpha+z-i \beta)^{s}}\right\} \\
& -\frac{1}{A^{s / 2}} \int_{0}^{\infty} d x\left\{\frac{1}{(x+\alpha+z+i \beta)^{s}}+\frac{1}{(x+\alpha+z-i \beta)^{s}}\right\} .
\end{aligned}
$$

We now note that, thanks to our choice for $\gamma$, collecting the second and the last terms under the integral and deriving with respect to $s$ in $s=0$ we obtain a vanishing term (see

\footnotetext{
${ }^{5}$ Abel-Plana formula is: $\sum_{n=0}^{\infty} f(n)=\frac{1}{2} f(0)+\int_{0}^{\infty} f(x) d x+i \int_{0}^{\infty} \frac{f(i x)-f(-i x)}{e^{2 \pi x}-1}$.
} 
Lemma 1 in [20]). The same happens for the first and the fourth term so that only the third term contribute to the derivative. Our conclusion is that

$$
\left.\frac{\partial}{\partial s}\right|_{s=0} \sigma_{k}(s ; z)=\left.\frac{\partial}{\partial s}\right|_{s=0}\left[\frac{1}{A^{s / 2}}\left(\zeta_{H}(\alpha+z+i \beta, s)+\zeta_{H}(\alpha+z-i \beta, s)\right)\right] .
$$

Using (5.63) we conclude that

$$
\left.\frac{\partial}{\partial s}\right|_{s=0} \zeta_{k}(s)=\left.\frac{\partial}{\partial s}\right|_{s=0} \hat{\zeta}_{k}(s)
$$

where

$$
\frac{1}{2} \hat{\zeta}_{k}(s):=\frac{\mathcal{T}}{2 \pi}\left[2 e E\left(\hat{\sigma}_{k}\left(s ;-\frac{1}{2}\right)-\frac{1}{2} \frac{1}{\mu_{k}^{2 s}}\right)+2 \int_{0}^{\infty} \hat{\sigma}_{k}(s ; z) d z\right],
$$

with

$$
\hat{\sigma}_{k}(s ; z)=\frac{1}{A^{s / 2}}\left(\zeta_{H}(\alpha+z+i \beta, s)+\zeta_{H}(\alpha+z-i \beta, s)\right) .
$$

Thus

$$
\begin{aligned}
\frac{1}{2} \hat{\zeta}_{k}(s)= & \frac{\mathcal{T}}{2 \pi}\left\{2 e E \frac{1}{A^{\frac{s}{2}}}\left[\zeta_{H}\left(\alpha-\frac{1}{2}+i \beta, s\right)+\zeta_{H}\left(\alpha-\frac{1}{2}-i \beta, s\right)\right]-e E \frac{1}{\mu_{k}^{2 s}}\right. \\
& \left.+2 \frac{1}{A^{\frac{s}{2}}} \frac{1}{s-1}\left[\zeta_{H}(\alpha+i \beta, s-1)+\zeta_{H}(\alpha-i \beta, s-1)\right]\right\} .
\end{aligned}
$$

Then we get (we momentarily omit the index $k$ from some formulas below)

$$
\begin{aligned}
\frac{1}{2} \zeta^{\prime}(0)=\frac{\mathcal{T}}{2 \pi}\{ & 2(e E)^{2} \log A-e E \log A+2 e E \log \frac{\Gamma(e E+i \beta) \Gamma(e E-i \beta)}{2 \pi} \\
& +e E \log \left(\mu_{k}^{2}\right)+(2+\log A)\left[\zeta_{H}(\alpha+i \beta,-1)+\zeta_{H}(\alpha-i \beta,-1)\right] \\
& \left.-2\left[\zeta_{H}^{\prime}(\alpha+i \beta,-1)+\zeta_{H}^{\prime}(\alpha-i \beta,-1)\right]\right\},
\end{aligned}
$$

where we used the well known relations

$$
\zeta_{H}(a, 0)=\frac{1}{2}-a, \quad \zeta_{H}^{\prime}(a, 0)=\log \frac{\Gamma(a)}{\sqrt{2 \pi}} .
$$

By going back to Lorentzian signature, through $e E \mapsto i e E$, we get

$$
\begin{aligned}
\frac{1}{2} \operatorname{Im} \zeta^{\prime}(0)= & \frac{\mathcal{T}}{2 \pi}\left[-e E \log A-e E \log \left(\Delta-(e E)^{2}\right)-e E \log (2 \cosh [2 \pi \sqrt{\Delta}]-2 \cosh [2 \pi e E])\right. \\
& \left.+e E \log \left(\mu_{k}^{2}\right)-2 \operatorname{Im}\left[\zeta_{H}^{\prime}(\alpha+i \beta,-1)+\zeta_{H}^{\prime}(\alpha-i \beta,-1)\right]\right] .
\end{aligned}
$$

To compute the last two terms we can start from (4.42) and use the identity

$$
-\frac{1}{2} \log ^{2}(-z)-\frac{\pi^{2}}{6}=\operatorname{Li}_{2}(z)+\operatorname{Li}_{2}(1 / z)
$$

to obtain the relation

$$
\operatorname{Im} \zeta_{H}^{\prime}\left(i \frac{x}{2}+\frac{1}{2} ;-1\right)=\frac{1}{8 \pi}\left[\operatorname{Li}_{2}\left(-e^{\pi x}\right)-\operatorname{Li}_{2}\left(-e^{-\pi x}\right)\right],
$$


and then

$$
\begin{aligned}
\frac{1}{2} \operatorname{Im} \zeta_{k}^{\prime}(0)=\frac{\mathcal{T}}{2 \pi}\{ & -e E \log (2 \cosh [2 \pi \sqrt{\Delta}]-2 \cosh [2 \pi e E]) \\
& -\frac{1}{4 \pi}\left[-\operatorname{Li}_{2}(-\exp [-2 \pi(\sqrt{\Delta}+e E)])+\operatorname{Li}_{2}(-\exp [2 \pi(\sqrt{\Delta}+e E)])\right. \\
& \left.\left.-\operatorname{Li}_{2}(-\exp [2 \pi(\sqrt{\Delta}-e E)])+\operatorname{Li}_{2}(-\exp [-2 \pi(\sqrt{\Delta}-e E)])\right]\right\} .
\end{aligned}
$$

Also in the Nariai case, the result coincides with the one obtained in the transmission coefficient approach. The calculation in the $\zeta$-function approach is much more difficult than the one in the transmission coefficient approach. Still, it furnishes the complete 1-loop effective action, and not simply its imaginary part.

\subsection{Instability of the thermal state}

Also in this case, according to the general discussion of section 2, we get

$$
\begin{aligned}
<N_{l}^{\text {out }}>_{\beta_{h}}= & \frac{1}{1+\exp \left[2 \pi\left(\omega-\varphi^{+}\right)\right]} \\
& +\frac{\cosh [2 \pi e E]+\cosh [2 \pi \omega]}{\cosh [2 \pi \sqrt{\Delta}]+\cosh [2 \pi \omega]} \frac{1}{2}\left(\tanh \left[\pi\left(\omega-\varphi^{+}\right)\right]+\tanh \left[\pi\left(|\omega|+\varphi^{-}\right)\right]\right)
\end{aligned}
$$

with $\varphi^{+}=2 e E=\varphi^{-}$. Thermality of the state affects the pair production induced by the presence of an electrostatic field, which is associated with the second term in (5.80). We recall that in terms of physical (dimensionful) variables, by taking into account that $T_{h}=\frac{\hbar c \sqrt{A}}{2 \pi k_{b}}$, and that $\omega_{\text {phys }}=\sqrt{A} \omega$, in such a way that $\beta_{\text {phys }} \omega_{\text {phys }}=2 \pi \omega$.

\section{Conclusions}

We have studied the spontaneous emission of charged Dirac particles by three special dS black hole solutions which share the relevant property to allow exact calculations and to present spherosymmetric metrics with a two-dimensional spherical part completely factorized (with at most a constant warping factor). As a consequence of the latter feature, the problem allows a reduction á la Kaluza-Klein to a two dimensional effective theory. This fact reflects itself in the common structure of our results in the $\zeta$-function approach as well as in the transmission coefficient approach, with the 4D imaginary part of the effective action appearing as a sum over $\mathrm{K}-\mathrm{K}$ modes of $2 \mathrm{D}$ terms. As to vacuum instability, our double check by the aforementioned approaches leads to identical results. Analogous results occur in the case of scalar fields on the same backgrounds [47]. Moreover, both the ultracold I case and the Nariai case make evident that calculations of the imaginary part of the effective action are easier in the transmission coefficient approach; still, it is to be pointed out that, a priori, the $\zeta$-function approach furnishes the complete 1-loop effective action (and then also vacuum polarization effects are taken into account [39]), and not only an evaluation of the vacuum instability (which is the only outcome of the transmission coefficient approach). It is also remarkable that the analysis of ultracold I and the Nariai cases points out that different regions in $\omega$ must be taken into account in 
the two approaches: in the transmission coefficient approach, only the level-crossing region is involved, instead in the $\zeta$-function approach the whole spectrum must be included.

As expected, the presence of an electrostatic field associated with the black hole charge induces a non-zero imaginary part of the effective action, i.e. to a vacuum instability which gives rise to the emission of charged pairs by the black hole, with a consequent discharge.

The ultracold II case is to some extent trivial, because it substantially reduces to the instability of the vacuum in a 2D Minkowski space with an homogeneous electrostatic field. This fact appears clearly by comparing our results e.g. with the ones for the $2 \mathrm{D}$ case in [41]. Nevertheless, the results obtained are meaningful, as we deal with the instability of a black hole with zero temperature.

The ultracold I case presents a less trivial structure, a non-homogeneous electrostatic field coupled with a Rindler-like horizon, and a non-zero temperature. Instability of the Boulware-like vacuum has been shown, and moreover also the instability of the HartleHawking thermal state has been displayed; general calculations carried out in section 2 show that the transmission coefficient which signals vacuum instability still plays a relevant role for a thermal state at finite temperature, and Thermofield Dynamics is in agreement with the results in [32].

The Nariai case is the most interesting one and also the most difficult, because it represents a charged black hole characterized by a finite spatial section and with an electrostatic field which reach a maximum between the black hole horizon and the cosmological event horizon (where it vanishes), and then in this respect it is really different from ReissnerNordström black holes (where the electrostatic field is mostly intense at the black hole horizon). Instability is again shown to occur.

For the given manifolds, a comparison between the exact results for the transmission coefficient and the WKB approximation ones obtained in [12] has also been performed, and, as expected, we have found that WKB is exact in the ultracold II case.

\section{References}

[1] G.W. Gibbons, Vacuum polarization and the spontaneous loss of charge by black holes, Commun. Math. Phys. 44 (1975) 245 [SPIRES].

[2] I.B. Khriplovich, Particle creation by charged black holes, Phys. Rept. 320 (1999) 37 [SPIRES].

[3] W. Heisenberg and H. Euler, Consequences of Dirac's theory of positrons, Z. Phys. 98 (1936) 714, English translation in [physics/0605038] [SPIRES].

[4] J.S. Schwinger, On gauge invariance and vacuum polarization, Phys. Rev. 82 (1951) 664 [SPIRES].

[5] S.P. Gavrilov, D.M. Gitman and J.L. Tomazelli, Density matrix of a quantum field in a particle-creating background, Nucl. Phys. B 795 (2008) 645 [hep-th/0612064] [SPIRES].

[6] S.P. Kim and D.N. Page, Schwinger pair production via instantons in a strong electric field, Phys. Rev. D 65 (2002) 105002 [hep-th/0005078] [SPIRES]; Schwinger pair production in electric and magnetic fields, Phys. Rev. D 73 (2006) 065020 [hep-th/0301132] [SPIRES]; 
Improved approximations for fermion pair production in inhomogeneous electric fields, Phys. Rev. D 75 (2007) 045013 [hep-th/0701047] [SPIRES]; Remarks on Schwinger pair production by charged black holes, Nuovo Cim. B 120 (2005) 1193 [gr-qc/0401057] [SPIRES].

[7] S.P. Kim, H.K. Lee and Y. Yoon, Effective action of scalar QED in electric field backgrounds, Phys. Rev. D 78 (2008) 105013 [arXiv:0807.2696] [SPIRES].

[8] T. Damour, Klein paradox and vacuum polarization, in Proceedings of First Marcel Grossmann Meeting on general relativity, Trieste Italy 1975, R. Ruffini ed., North-Holland, Amsterdam The Netherlands (1977) pg. 459.

[9] N. Deruelle, Classical and quantum states in black hole physics, in Proceeding of First Marcel Grossmann meeting on general relativity, Trieste Italy 1975, R. Ruffini ed., North-Holland, Amsterdam The Netherlands (1977), pg. 483.

[10] N. Deruelle and R. Ruffini, Quantum and classical relativistic energy states in stationary geometries, Phys. Lett. B 52 (1974) 437 [SPIRES].

[11] F. Belgiorno and S.L. Cacciatori, Quantum effects for the Dirac field in Reissner-Nordstrom-Ads black hole background, Class. Quant. Grav. 25 (2008) 105013 [arXiv:0710.2014] [SPIRES].

[12] F. Belgiorno and S.L. Cacciatori, Massive Dirac particles on the background of charged de-Sitter black hole manifolds, Phys. Rev. D 79 (2009) 124024 [arXiv:0810.1642] [SPIRES].

[13] L.J. Romans, Supersymmetric, cold and lukewarm black holes in cosmological Einstein-Maxwell theory, Nucl. Phys. B 383 (1992) 395 [hep-th/9203018] [SPIRES].

[14] R. Bousso, Quantum global structure of de Sitter space, Phys. Rev. D 60 (1999) 063503 [hep-th/9902183] [SPIRES].

[15] R.B. Mann and S.F. Ross, Cosmological production of charged black hole pairs, Phys. Rev. D 52 (1995) 2254 [gr-qc/9504015] [SPIRES].

[16] T. Kaluza, On the problem of unity in physics, Sitzungsber. Preuss. Akad. Wiss. Berlin (Math. Phys.) 1921 (1921) 966 [SPIRES].

[17] O. Klein, Quantum theory and five-dimensional theory of relativity, Z. Phys. 37 (1926) 895 [Surveys High Energ. Phys. 5 (1986) 241] [SPIRES].

[18] M.J. Duff, B.E.W. Nilsson and C.N. Pope, Kaluza-Klein supergravity, Phys. Rept. 130 (1986) 1 [SPIRES].

[19] A. Salam and E. Sezgin, Compactification, in Supergravities in diverse dimensions, vol. 2, A. Salam and E. Sezgin eds., North-Holland, Amsterdam The Netherlands (1989), pg. 1239-1281, (see Book Index).

[20] S.L. Cacciatori, On a polynomial zeta function, arXiv:0902.3190 [SPIRES].

[21] E. Brézin and C. Itzykson, Pair production in vacuum by an alternating field, Phys. Rev. D 2 (1970) 1191 [SPIRES].

[22] C. Itzykson and J.-B. Zuber, Quantum field theory, McGraw Hill, New York U.S.A. (1980) [SPIRES]

[23] A.I. Nikishov, Pair production by a constant external field, Sov. Phys. JETP 30 (1970) 660.

[24] N.B. Narozhnyi and A.I. Nikishov, The Simplest processes in the pair creating electric field, Yad. Fiz. 11 (1970) 1072 [Sov. J. Nucl. Phys. 11 (1970) 596] [SPIRES]. 
[25] S.P. Gavrilov, D.M. Gitman and J.L. Tomazelli, Density matrix of a quantum field in a particle-creating background, Nucl. Phys. B 795 (2008) 645 [hep-th/0612064] [SPIRES].

[26] W. Israel, Thermo field dynamics of black holes, Phys. Lett. A 57 (1976) 107 [SPIRES].

[27] W.G. Unruh, Notes on black hole evaporation, Phys. Rev. D 14 (1976) 870 [SPIRES].

[28] Y. Takahashi and H. Umezawa, Thermo field dynamics, Collective Phenomena 2 (1975) 55.

[29] H. Umezawa, H. Matsumoto and M. Tachiki, Thermo field dynamics and condensed states, North-Holland Publishing Company, Amsterdam The Netherlands (1982).

[30] H. Umezawa, Advanced field theory. Micro, macro, and thermal physics, American Institute of Physics, New York U.S.A. (1993).

[31] U. Moschella and R. Schaeffer, A note on canonical quantization of fields on a manifold, JCAP 02 (2009) 033 [arXiv: 0802 .2447] [SPIRES].

[32] S.P. Kim, H.K. Lee and Y. Yoon, Schwinger pair production at finite temperature in QED, Phys. Rev. D 79 (2009) 045024 [arXiv:0811.0349] [SPIRES].

[33] S.P. Kim and H.K. Lee, Schwinger pair production at finite temperature in scalar QED, Phys. Rev. D 76 (2007) 125002 [arXiv:0706.2216] [SPIRES].

[34] S.P. Gavrilov and D.M. Gitman, Energy-momentum tensor in thermal strong-field QED with unstable vacuum, J. Phys. A 41 (2008) 164046 [arXiv:0710.3933] [SPIRES].

[35] A. Das, Finite temperature field theory, World Scientific Publishing Company, Singapore Republic of Singapore (1997).

[36] F.C. Khanna, A.P.C. Malbouisson, J.M.C. Malbouisson, and A.E. Santana, Thermal quantum field theory, World Scientific Publishing Company, Singapore Republic of Singapore (2009).

[37] D.N. Page, Particle emission rates from a black hole. 3. Charged leptons from a nonrotating hole, Phys. Rev. D 16 (1977) 2402 [SPIRES].

[38] M. Abramowitz and I.A. Stegun, Handbook of mathematical functions with formulas, graphs and mathematical tables, Dover Publications Inc., New York U.S.A. (1965).

[39] S.K. Blau, M. Visser and A. Wipf, Analytical results for the effective action, Int. J. Mod. Phys. A 6 (1991) 5409 [arXiv:0906 . 2851] [SPIRES].

[40] P.B. Gilkey, Invariance theory, the heat equation and the Atiyah-Singer index theorem, CRC press, Boca Raton U.S.A. (1995).

[41] Q.-g. Lin, Electron positron pair creation in vacuum by an electromagnetic field in $3+1$ and lower dimensions, J. Phys. G 25 (1999) 17 [hep-th/9810037] [SPIRES].

[42] V.B. Adesi and S. Zerbini, Analytic continuation of the Hurwitz zeta function with physical application, J. Math. Phys. 43 (2002) 3759 [hep-th/0109136] [SPIRES].

[43] C.A. Manogue, The Klein paradox and superradiance, Ann. Phys. 181 (1988) 261.

[44] R. Brout, S. Massar, S. Popescu, R. Parentani and P. Spindel, Quantum back reaction on a classical field, Phys. Rev. D 52 (1995) 1119 [hep-th/9311019] [SPIRES].

[45] C. Gabriel and P. Spindel, Quantum charged fields in Rindler space, Annals Phys. 284 (2000) 263 [gr-qc/9912016] [SPIRES].

[46] J.G. Russo, On Schwinger pair creation in gravity and in closed superstring theory, JHEP 03 (2009) 080 [arXiv:0901.1664] [SPIRES].

[47] F Belgiorno, S.L. Cacciatori and F. Dalla Piazza, Quantum instability for charged scalar particles on charged Nariai and ultracold black hole manifolds. 